\title{
Phosphine-Free Palladium Catalyzed C-H Bond Arylation of Free (N-H)-Indoles and Pyrroles
}

\author{
Xiang Wang, Denis V. Gribkov, Dalibor Sames* \\ Department of Chemistry, Columbia University, 3000 Broadway, New York, New York, 10027
}

\section{Supporting Information}

Material and general methods 
Materials and General Methods. All arylation reactions were carried out in closed glass vials (VWR, $8 \mathrm{~mL}$ ) equipped with a cap (Teflon septum, Kimble Glass), and heated in a 34-well reaction block (Chemglass). Elemental Analyses were performed by Schwarzkopf Microanalytical Laboratory, Inc, Woodside, NY. High resolution mass spectrometry analyses were conducted by Dr. Itagaki at Columbia University. NMR spectra were obtained on a Bruker spectrometer operating at $400 \mathrm{MHz}$ or $300 \mathrm{MHz}$ for ${ }^{1} \mathrm{H}$ NMR, $100 \mathrm{MHz}$ or $75 \mathrm{MHz}$ for ${ }^{13} \mathrm{C} \mathrm{NMR}$ in $\mathrm{CDCl}_{3}$ or DMSO-d . The spectra were referenced to the solvent residual peak $\left(\mathrm{CDCl}_{3}: 7.26 \mathrm{ppm}\right.$ in ${ }^{1} \mathrm{H} \mathrm{NMR}$ and $77.0 \mathrm{ppm}$ in ${ }^{13} \mathrm{C}$ NMR; DMSO- $d_{6}: 2.50 \mathrm{ppm}$ in ${ }^{1} \mathrm{H}$ NMR and $39.5 \mathrm{ppm}$ in $\left.{ }^{13} \mathrm{C} \mathrm{NMR}\right)$ or TMS (CDCl $\left.3,{ }^{1} \mathrm{H} \mathrm{NMR}\right)$. IR spectra were obtained on a Bomen MB-100 FT IR spectrometer. Gas chromatography was performed on an Agilent Technologies 6890N gas chromatograph equipped with a $25 \mathrm{~m}$ polydimethylsiloxane capillary column and 5973 Mass Selective Detector. Flash column chromatography was performed employing 200-400 mesh silica gel (EM). CsOAc (Strem) was dried (see below) and stored in a drybox, and small batches $(<5 \mathrm{~g})$ that were used for reaction were stored in a benchtop desiccator outside drybox. $\mathrm{Pd}(\mathrm{OAc})_{2}$ (Strem), $N, N$-dimethylacetamide (DMA, Anhydrous, Aldrich), haloarenes (Aldrich) and all other reagents were obtained commercially and used as received. $N^{9}$-acetyltryptamine, ${ }^{1} N^{9}$-Boc-tryptamine, ${ }^{2}$ 2-phenyl-5-(trimethylacetylamido)indol, ${ }^{3} N$-(4-iodophenyl)pivalamide, ${ }^{4}$ 1-acetamido-3-iodobenzene, ${ }^{5} N$-(thiazol-2-yl)pivalamide ${ }^{6}$ and 2-phenylpyrrole ${ }^{3}$ were synthesized via standard methods described in literature.

Procedure for drying CsOAc: A solution of CsOAc (Strem, $25 \mathrm{~g}$ ), $\mathrm{H}_{2} \mathrm{O}$ (10 mL) (added for dissolution purpose) and HOAc $(10 \mathrm{~mL})$ was stirred at $80{ }^{\circ} \mathrm{C}$ for 5 min before the solvent was evaporated through a rotavap (Drying CsOAc in the absence of HOAc results in formation of a small amount of $\mathrm{CsOH}$, which leads to lower conversion and selectivity). The resulting slurry material was then heated at $100{ }^{\circ} \mathrm{C}$ under high vacuum (50 mtorr) for $4 \mathrm{~h}$ to give a free-flowing fine powder, which was stored in an Argon-filled drybox. Small batches of CsOAc $(<5 \mathrm{~g})$ were stored in a benchtop desiccator for reaction usage.

Typical procedure for arylation of free (N-H)-indole with iodoarenes (Procedure A): On benchtop, CsOAc (260 mg, $1.35 \mathrm{mmol})$ was placed in a vial containing indole $(58.5 \mathrm{mg}, 0.50$ 
mmol). The vial was capped with a teflon septum cap, evacuated and refilled with argon via a needle through the septum. The evacuation/refill procedure was repeated twice before $\mathrm{PhI}(143 \mathrm{mg}$, $0.70 \mathrm{mmol})$ and a solution of $\mathrm{Pd}(\mathrm{OAc})_{2}(100 \mu \mathrm{L}, 11.0 \mathrm{mg} / \mathrm{mL}$ in DMA $)$ were added sequentially via syringe. Under an argon purge provided by a needle, the septum cap was replaced with a FTFEfaced Kimble phenolic cap. The reaction mixture was then stirred at $125{ }^{\circ} \mathrm{C}$, at which point the base melted and the reaction mixture became homogenous. The resulting solution was heated for $24 \mathrm{~h}$ while white precipitate (CsI) started to form. The reaction mixture was then cooled to room temperature, diluted with EtOAc $(5 \mathrm{~mL})$, stirred for $1 \mathrm{~min}$, passed through a short plug of silica gel, and eluted with EtOAc $(15 \mathrm{~mL})$. The resulting solution was concentrated, absorbed on silica gel ( 2g), dry-loaded and chromatographed $\left(\mathrm{SiO}_{2}, 15: 1\right.$ hexanes:EtOAc) to afford 2-phenylindole (64 $\mathrm{mg}, 66 \%)$ as a white solid.

\section{Typical procedure for arylation of C-3 substituted indole with bromoarenes (Procedure}

B): $\operatorname{CsOAc}(260 \mathrm{mg}, 1.35 \mathrm{mmol})$ was placed in a vial containing 3-methylindole $(65.5 \mathrm{mg}, 0.50$ mmol). The vial was capped with a teflon septum cap, evacuated, and refilled with argon via a needle through the septum. The evacuation/refill procedure was repeated twice. $\mathrm{PhBr}(110 \mathrm{mg}, 0.7$ $\mathrm{mmol})$, diisopropylamine $(50 \mathrm{mg}, 0.50 \mathrm{mmol})$, and a solution of $\mathrm{Pd}(\mathrm{OAc})_{2}(100 \mu \mathrm{L}, 11.0 \mathrm{mg} / \mathrm{mL}$ in DMA) were added sequentially via syringe. Under an argon purge provided by a needle, the septum cap was replaced with a FTFE-faced Kimble phenolic cap. The solid mixture was then heated at 125 ${ }^{\circ} \mathrm{C}$, at which point the base melted and the reaction mixture became homogenous. The reaction mixture was heated for $24 \mathrm{~h}$ before being cooled to room temperature. After addition of EtOAc $(5 \mathrm{~mL})$, the reaction mixture was stirred for $1 \mathrm{~min}$, passed through a short plug of silica gel, and eluted with EtOAc $(15 \mathrm{~mL})$. The resulting solution was concentrated, absorbed on silica gel $(\sim 2 \mathrm{~g})$, dry-loaded and chromatographed ( $\mathrm{SiO}_{2}, 20: 1$ hexanes:EtOAc) to afford 3-methyl-2-phenylindole (65 $\mathrm{mg}, 63 \%$ ) as a white solid. Unreacted 3-methylindole could be recovered by column chromatography.

Note: The arylation reactions described above demonstrated good tolerance toward moisture and air. The benchtop procedure described herein (evacuation/refill of argon via needle) gave results similar to reactions that were set up in a glovebox. 
Effect of Ligand, Base, and Solvent. Selected examples. ${ }^{a}$

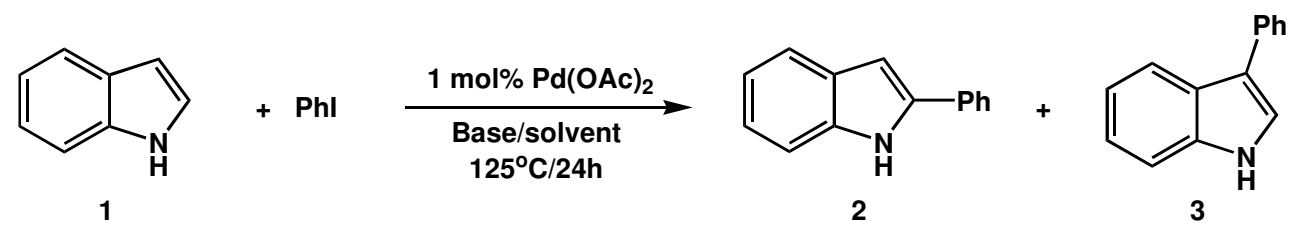

\begin{tabular}{|c|c|c|c|c|}
\hline Entry & Ligand (mol\%) & Base & Yield(\%) ${ }^{b}$ & Ratio $(2 / 3)^{c}$ \\
\hline $1^{d}$ & $\mathrm{PPh}_{3}(4)$ & CsOAc & 6 & $5: 1$ \\
\hline 2 & $\mathrm{PPh}_{3}(4)$ & CsOAc & 16 & $9: 1$ \\
\hline 3 & $\mathrm{PPh}_{3}(2)$ & CsOAc & 23 & $9: 1$ \\
\hline 4 & $\mathrm{PPh}_{3}(1)$ & CsOAc & 68 & $15: 1$ \\
\hline 5 & $\operatorname{IPr}(2)$ & CsOAc & 70 & $15: 1$ \\
\hline 6 & $\mathrm{HPC}_{3} \mathrm{BF}_{4}(2)$ & CsOAc & 54 & $14: 1$ \\
\hline 7 & 2-Di(t-butylphosphino)biphenyl (2) & CsOAc & 3 & - \\
\hline 8 & -- & CsOAc & 75 & $18: 1$ \\
\hline $9^{e}$ & -- & CsOAc & 56 & $9: 1$ \\
\hline 10 & -- & AgOAc & $<2$ & -- \\
\hline $11^{e}$ & -- & AgOAc & $<2$ & -- \\
\hline
\end{tabular}

${ }^{a}$ Conditions: Indole (5 M), PhI (1.2 equiv.), CsOAc (2.8 equiv.), DMA, $125{ }^{\circ} \mathrm{C} / 24 \mathrm{~h} .{ }^{b} \mathrm{GC}$ yield calibrated against an internal standard (dodecane). ${ }^{c}$ Ratio in crude reaction mixture determined by GC analysis. ${ }^{d}$ Indole $(1.25 \mathrm{M})$ used. ${ }^{e} \mathrm{HOAc}$ was used instead of DMA.

\section{Spectroscopic characterization of products and elemental analysis/HRMS of new compounds}

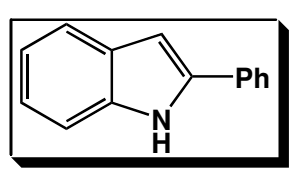

2-Phenylindole (Table 2, entry 1$).^{7}$ Procedure $\mathrm{A}, \quad 66 \% \quad\left(\mathrm{SiO}_{2}, \quad 15: 1\right.$ hexanes:EtOAc): ${ }^{1} \mathrm{H}$ NMR $\left(400 \mathrm{MHz}, \mathrm{DMSO}-d_{6}\right) \delta 11.6(\mathrm{~s}, 1 \mathrm{H}), 7.87(\mathrm{dd}, J=$ $1.2,8.4 \mathrm{~Hz}, 2 \mathrm{H}), 7.53(\mathrm{~d}, J=7.6 \mathrm{~Hz}, 1 \mathrm{H}), 7.46(\mathrm{t}, J=7.6 \mathrm{~Hz}, 2 \mathrm{H}), 7.41$ (dd, $J=$ $0.8,8.0 \mathrm{~Hz}, 1 \mathrm{H}), 7.29-7.33(\mathrm{~m}, 1 \mathrm{H}), 7.08-7.13(\mathrm{~m}, 1 \mathrm{H}), 7.00(\mathrm{dt}, J=0.8,8.0$ $\mathrm{Hz}, 1 \mathrm{H}), 6.90(\mathrm{~m}, 1 \mathrm{H}) ;{ }^{13} \mathrm{C}$ NMR $\left(100 \mathrm{MHz}, \mathrm{DMSO}-d_{6}\right) \delta 137.6,137.1,132.2,128.8,128.6$, 127.3, 124.9, 121.5, 120.0, 119.3, 111.3, 98.6.

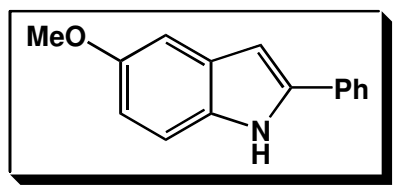

5-Methoxy-2-phenylindole (Table 2, entry 2$).{ }^{8}$ Procedure $\mathrm{A}, 64 \%\left(\mathrm{SiO}_{2}\right.$, 10:1 to 8:1 hexanes:EtOAc): ${ }^{1} \mathrm{H}$ NMR $\left(300 \mathrm{MHz}\right.$, DMSO- $\left.d_{6}\right) \delta 11.34$ (s, 1 H), 7.81-7.83 (m, $2 \mathrm{H}), 7.43(\mathrm{t}, J=7.6 \mathrm{~Hz}, 2 \mathrm{H}), 7.26-7.31(\mathrm{~m}, 2 \mathrm{H}), 7.02$ $(\mathrm{d}, J=2.4 \mathrm{~Hz}, 1 \mathrm{H}), 6.79(\mathrm{~d}, J=1.5 \mathrm{~Hz}, 1 \mathrm{H}), 6.75(\mathrm{dd}, J=2.4,8.8 \mathrm{~Hz}, 1$ $\mathrm{H}), 3.76(\mathrm{~s}, 3 \mathrm{H}) ;{ }^{13} \mathrm{C}$ NMR $\left(75 \mathrm{MHz}\right.$, DMSO- $\left.d_{6}\right) \delta 153.6,138.1,132.3,129.0,128.8,127.2,124.8$, $111.9,111.8,101.6,98.5,55.2$. One carbon peak in aromatic region is missing in our assignment, presumably due to overlap.

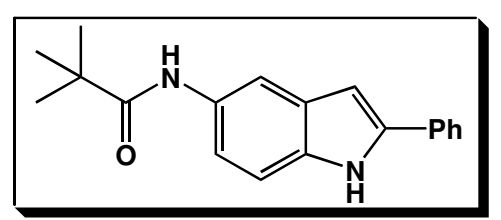

2-Phenyl-5-(trimethylacetylamido)indole (Table 2, entry 3). Procedure A, $62 \%\left(\mathrm{SiO}_{2}, 3: 1\right.$ to 2:1 hexanes:EtOAc, followed by rechromatography under same conditions): ${ }^{1} \mathrm{H}$ NMR $(400 \mathrm{MHz}$, DMSO- $\left.d_{6}\right) \delta 11.4(\mathrm{~s}, 1 \mathrm{H}), 9.02(\mathrm{~s}, 1 \mathrm{H}), 7.81-7.85(\mathrm{~m}, 2 \mathrm{H}), 7.45(\mathrm{t}, J$ 
$=7.5 \mathrm{~Hz}, 1 \mathrm{H}), 7.23-7.31(\mathrm{~m}, 3 \mathrm{H}), 6.85(\mathrm{~d}, J=1.2 \mathrm{~Hz}, 1 \mathrm{H}), 1.26(\mathrm{~s}, 9 \mathrm{H}) ;{ }^{13} \mathrm{C} \mathrm{NMR}(100 \mathrm{MHz}$, DMSO- $\left.d_{6}\right): \delta 175.9,138.0,134.0,132.2,131.6,128.8,128.3,127.3,124.9,116.9,112.1,110.7$, 98.7, 27.4. IR (film, $\mathrm{cm}^{-1}$ ): 1620, 1542, 1480. HRMS for $\mathrm{C}_{19} \mathrm{H}_{21} \mathrm{~N}_{2} \mathrm{O}\left(\mathrm{MH}^{+}\right.$) calcd (found): 293.1654 (293.1646).

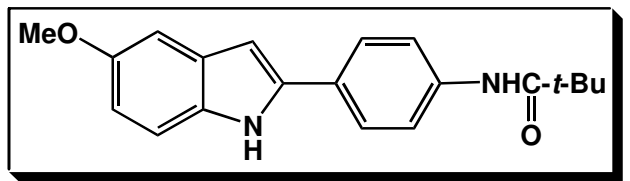

5-Methoxy-2-(4-trimethylacetylamidiphenyl)indole (Table 2, entry 4). Procedure A, 53\% $\left(\mathrm{SiO}_{2}, 3: 1\right.$ to $2: 1$ hexanes:EtOAc): ${ }^{1} \mathrm{H}$ NMR (300 MHz, DMSO- $\left.d_{6}\right) \delta 11.3(\mathrm{~s}$, $1 \mathrm{H}), 9.3(\mathrm{~s}, 1 \mathrm{H}), 7.76(\mathrm{~s}, 4 \mathrm{H}), 7.26(\mathrm{~d}, J=9.0 \mathrm{~Hz}, 1 \mathrm{H})$, $6.90(\mathrm{~d}, J=2.1 \mathrm{~Hz}, 1 \mathrm{H}), 6.73-6.74(\mathrm{~m}, 2 \mathrm{H}), 3.76(\mathrm{~s}, 3 \mathrm{H}), 1.25(\mathrm{~s}, 9 \mathrm{H}) ;{ }^{13} \mathrm{C} \mathrm{NMR}(75 \mathrm{MHz}$, DMSO- $\left.d_{6}\right) \delta 176.5,153.6,138.6,138.2,132.1,129.2,127.2,126.1,125.0,120.4,111.8,111.4$, 101.4, 97.8, 55.2, 27.2. IR (film, $\mathrm{cm}^{-1}$ ): 2964, 1662, 1514, 1482, 1455, 1217, 1161. HRMS for $\mathrm{C}_{20} \mathrm{H}_{22} \mathrm{~N}_{2} \mathrm{O}_{2}\left(\mathrm{M}^{+}\right)$calcd (found): 322.1681 (322.1677).

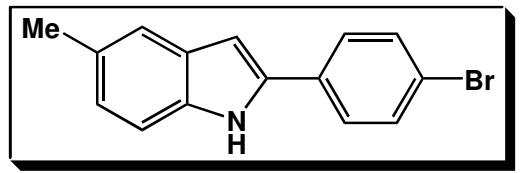

2-(4-Bromophenyl)-5-methylindole (Table 2, entry 5). Procedure A, $44 \%\left(\mathrm{SiO}_{2}, 15: 1\right.$ to $12: 1$ hexanes:EtOAc): ${ }^{1} \mathrm{H}$ NMR $(300 \mathrm{MHz}$, DMSO-d 6$) \delta 11.5(\mathrm{~s}, 1 \mathrm{H}), 7.78(\mathrm{~d}, J=8.4 \mathrm{~Hz}, 2 \mathrm{H}), 7.63(\mathrm{~d}, J=8.7$ $\mathrm{Hz}, 2 \mathrm{H}), 7.27-7.30$ (m, $2 \mathrm{H}), 6.94(\mathrm{~d}, J=8.4 \mathrm{~Hz}, 1 \mathrm{H}), 6.82$ (s, 1 $\mathrm{H}), 2.32(\mathrm{~s}, 3 \mathrm{H}) ;{ }^{13} \mathrm{C}$ NMR $\left(75 \mathrm{MHz}, \mathrm{DMSO}-d_{6}\right) \delta 136.3,135.6,131.8,131.6,128.8,128.0,126.8$, 123.6, 120.1, 119.8, 111.1, 98.9, 21.2. IR (film, $\mathrm{cm}^{-1}$ ): 3446, 1418, 828, 800. Elemental analysis for $\mathrm{C}_{15} \mathrm{H}_{12} \mathrm{BrN}$ calcd (found): C, 62.96 (63.15); H, 4.23 (4.18); N, 4.89 (4.77).

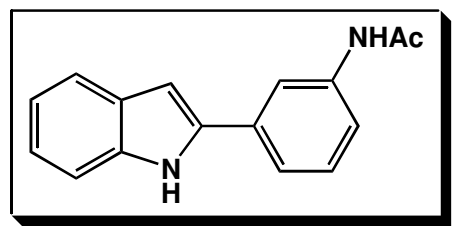

2-(3-Acetylamidophenyl)indole (Table 2, entry 6). Procedure A, 23\% $\left(\mathrm{SiO}_{2}, 1: 1\right.$ to $3: 2$ EtOAc:hexanes): ${ }^{1} \mathrm{H} \mathrm{NMR}\left(300 \mathrm{MHz}, \mathrm{DMSO}-d_{6}\right) \delta$ 11.5 (s, $1 \mathrm{H}), 10.0(\mathrm{~s}, 1 \mathrm{H}), 8.09$ (s, $1 \mathrm{H}), 7.38-7.53(\mathrm{~m}, 5 \mathrm{H}), 7.08-7.12$ (m, $1 \mathrm{H}), 6.98-7.02(\mathrm{~m}, 1 \mathrm{H}), 6.77(\mathrm{~s}, 1 \mathrm{H}), 2.10(\mathrm{~s}, 3 \mathrm{H}) ;{ }^{13} \mathrm{C}$ NMR $(75$ $\left.\mathrm{MHz}, \mathrm{DMSO}-d_{6}\right) \delta 168.4,139.8,137.7,137.1,132.7,129.2,128.5$, 121.5, 120.0, 119.9, 119.3, 118.4, 116.0, 111.4, 98.6, 24.0. IR (film, $\mathrm{cm}^{-1}$ ): 2360, 2342, 1671, 1610, 1525, 1453, 1299, 781. HRMS for $\mathrm{C}_{16} \mathrm{H}_{14} \mathrm{~N}_{2} \mathrm{O}\left(\mathrm{M}^{+}\right)$calcd (found): 250.1106 (250.1100).

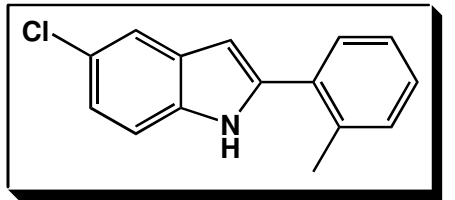

5-Chloro-2-(2-tolyl)indole (Table 2, entry 7). Procedure $\mathrm{A}, 60 \%\left(\mathrm{SiO}_{2}\right.$, 12:1 to 10:1 hexanes:EtOAc): ${ }^{1} \mathrm{H}$ NMR $\left(300 \mathrm{MHz}, \mathrm{CDCl}_{3}\right) \delta 8.07$ (s, 1 $\mathrm{H}), 7.64(\mathrm{~d}, J=1.8 \mathrm{~Hz}, 1 \mathrm{H}), 7.44-7.47(\mathrm{~m}, 1 \mathrm{H}), 7.29-7.35(\mathrm{~m}, 4 \mathrm{H})$, $7.19(\mathrm{dd}, J=1.8,7.6 \mathrm{~Hz}, 1 \mathrm{H}), 6.57-6.58(\mathrm{~m}, 1 \mathrm{H}), 2.51(\mathrm{~s}, 3 \mathrm{H}) ;{ }^{13} \mathrm{C}$ NMR $\left(75 \mathrm{MHz}, \mathrm{CDCl}_{3}\right) \delta 138.8,136.1,134.3,132.0,131.1,129.9,128.9,128.3,126.1,125.6$, 122.2, 119.8, 111.7, 102.5, 21.0. IR (film, $\left.\mathrm{cm}^{-1}\right): 3420,1491,792,758$. Elemental analysis for $\mathrm{C}_{15} \mathrm{H}_{12} \mathrm{ClN}\left(\mathrm{M}^{+}\right)$calcd (found): C, 74.53 (74.81); H, 5.00 (4.86); N, 5.79 (5.75).

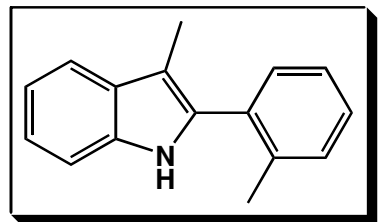

3-Methyl-2-(2-tolyl)indole (Table 2, entry 8). Procedure A, 28\% $\left(\mathrm{SiO}_{2}\right.$, 25:1 hexanes:EtOAc): ${ }^{1} \mathrm{H}$ NMR $\left(300 \mathrm{MHz}, \mathrm{CDCl}_{3}\right) \delta 7.76$ (bs, $\left.1 \mathrm{H}\right), 7.58$ $(\mathrm{d}, J=7.6 \mathrm{~Hz}, 1 \mathrm{H}), 7.11-7.33(\mathrm{~m}, 7 \mathrm{H}), 2.27(\mathrm{~s}, 3 \mathrm{H}), 2.20(\mathrm{~s}, 3 \mathrm{H}) ;{ }^{13} \mathrm{C}$ NMR $\left(75 \mathrm{MHz}, \mathrm{CDCl}_{3}\right): \delta 137.6,135.6,134.2,132.6,130.8,130.3,129.1$, $128.3,125.6,121.8,119.3,118.8,110.5,109.3,20.0,9.2$. IR (film, $\mathrm{cm}^{-1}$ ): 1652, 1583, 1520, 1451, 756, 732. HRMS for $\mathrm{C}_{16} \mathrm{H}_{15} \mathrm{~N}\left(\mathrm{M}^{+}\right)$calcd (found): 221.1204 (221.1199). 


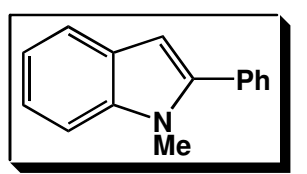

$N$-Methyl-2-phenylindole (Table 2, entry 9). ${ }^{9}$ Procedure A, $75 \%\left(\mathrm{SiO}_{2}, 35: 1\right.$ hexanes:EtOAc): ${ }^{1} \mathrm{H}$ NMR $\left(400 \mathrm{MHz}, \mathrm{CDCl}_{3}\right) \delta$ 7.70-7.72 (m, $\left.1 \mathrm{H}\right), 7.56-7.59$ (m, $2 \mathrm{H}), 7.51-7.55$ (m, $2 \mathrm{H}), 7.41-7.48$ (m, $2 \mathrm{H}), 7.29-7.34$ (m, $1 \mathrm{H}), 7.20-7.24$ $(\mathrm{m}, 1 \mathrm{H}), 6.61(\mathrm{~s}, 1 \mathrm{H}), 3.80(\mathrm{~s}, 3 \mathrm{H}) ;{ }^{13} \mathrm{C} \mathrm{NMR}\left(100 \mathrm{MHz}, \mathrm{CDCl}_{3}\right) \delta 141.5$, $138.3,132.8,129.3,128.5,128.0,127.8,121.6,120.5,119.8,109.6,101.6,31.1$.

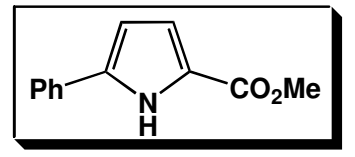

2-carbomethoxy-4-phenylpyrrole (Table 2, entry 10$).{ }^{10}$ Procedure A, 30\% $\left(\mathrm{SiO}_{2}, 12: 1\right.$ to $10: 1$ to $8: 1$ hexanes:EtOAc): ${ }^{1} \mathrm{H} \mathrm{NMR}\left(300 \mathrm{MHz}, \mathrm{CDCl}_{3}\right) \delta$ 9.70 (s, $1 \mathrm{H}), 7.60-7.63$ (m, $2 \mathrm{H}), 7.44-7.58$ (m, $2 \mathrm{H}), 7.30-7.33$ (m, $1 \mathrm{H}), 6.98$ $(\mathrm{dd}, J=2.4,3.9 \mathrm{~Hz}, 1 \mathrm{H}), 6.52(\mathrm{dd}, J=2.7,3.6 \mathrm{~Hz}, 1 \mathrm{H}), 3.88(\mathrm{~s}, 3 \mathrm{H}) ;{ }^{13} \mathrm{C} \mathrm{NMR}\left(75 \mathrm{MHz}, \mathrm{CDCl}_{3}\right)$ $\delta 161.8,137.1,131.4,128.9,127.7,124.9,123.0,116.9,108.0,51.6$. Elemental analysis for $\mathrm{C}_{12} \mathrm{H}_{11} \mathrm{NO}_{2}$ calcd (found): $\mathrm{C}, 71.63$ (71.66); $\mathrm{H}, 5.51$ (5.41); N, 6.96 (6.87).

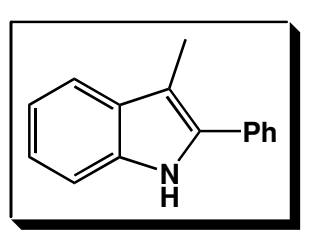

3-Methyl-2-phenylindole (Table 3, entry 1$).{ }^{11}$ Procedure $\mathrm{B}, 63 \%\left(\mathrm{SiO}_{2}, 20: 1\right.$ hexanes:EtOAc): ${ }^{1} \mathrm{H}$ NMR $\left(300 \mathrm{MHz}, \mathrm{CDCl}_{3}\right) \delta 7.99(\mathrm{bs}, 1 \mathrm{H}), 7.56-7.61(\mathrm{~m}, 3$ $\mathrm{H}), 7.47(\mathrm{t}, J=7.8 \mathrm{~Hz}, 2 \mathrm{H}), 7.33-7.37(\mathrm{~m}, 2 \mathrm{H}), 7.20(\mathrm{td}, J=1.3,7.5 \mathrm{~Hz}, 1 \mathrm{H})$, $7.14(\mathrm{tm}, J=7.3 \mathrm{~Hz}, 1 \mathrm{H}), 2.46(\mathrm{~s}, 3 \mathrm{H}) ;{ }^{13} \mathrm{C} \mathrm{NMR}\left(75 \mathrm{MHz}, \mathrm{CDCl}_{3}\right) \delta 135.7$, $133.9,133.2,129.9,128.7,127.7,127.2,122.2,119.5,118.9,110.7,108.6,9.6$.

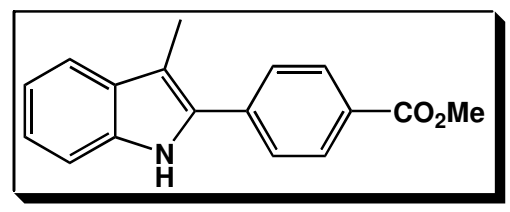

2-(4-Methoxycarbonylphenyl)-3-methylindole (Table 3, entry 2). Procedure $\mathrm{B}, 74 \%\left(\mathrm{SiO}_{2}, 8: 1\right.$ gradient to $4: 1$ hexanes:EtOAc): ${ }^{1} \mathrm{H}$ NMR $\left(300 \mathrm{MHz}\right.$, DMSO- $\left.d_{6}\right) \delta 11.3(\mathrm{~s}, 1 \mathrm{H}), 8.09(\mathrm{~d}, J=8.4 \mathrm{~Hz}, 2$ H), $7.84(\mathrm{~d}, J=8.4 \mathrm{~Hz}, 2 \mathrm{H}), 7.57(\mathrm{~d}, J=7.8 \mathrm{~Hz}, 1 \mathrm{H}), 7.41(\mathrm{~d}, J=$ $7.8 \mathrm{~Hz}, 1 \mathrm{H}), 7.16(\mathrm{t}, J=7.5 \mathrm{~Hz}, 1 \mathrm{H}), 7.04(\mathrm{t}, J=7.5 \mathrm{~Hz}, 1 \mathrm{H}), 3.88(\mathrm{~s}, 3 \mathrm{H}), 2.47(\mathrm{~s}, 3 \mathrm{H}) ;{ }^{13} \mathrm{C}$ NMR $\left(75 \mathrm{MHz}\right.$, DMSO- $\left.d_{6}\right) \delta 166.0,137.6,136.3,132.3,129.5,129.3,127.4,127.2,122.3,118.8$, $111.2,108.9,52.0,10.0$. One carbon peak in aromatic region is missing in our assignment, presumably due to overlap. IR (film, $\mathrm{cm}^{-1}$ ): $1690,1070,1025$. Elemental analysis for $\mathrm{C}_{17} \mathrm{H}_{15} \mathrm{NO}_{2}$ calcd (found): C, 76.96 (76.77); H, 5.70 (5.60); N, 5.28 (5.16).

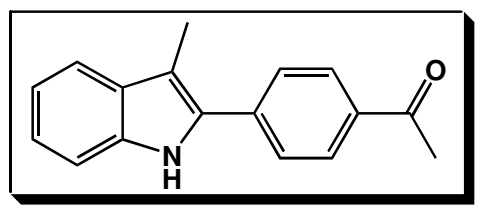

2-(4-acetylphenyl)-3-methylindole (Table 3, entry 3). Procedure B, 70\% ( $\mathrm{SiO}_{2}, 5: 1$ hexanes:EtOAc): ${ }^{1} \mathrm{H}$ NMR $\left(300 \mathrm{MHz}, \mathrm{CDCl}_{3}\right) \delta 8.19$ (br s, $1 \mathrm{H}), 8.03(\mathrm{~d}, J=8.4 \mathrm{~Hz}, 2 \mathrm{H}), 7.67-7.61(\mathrm{~m}, 3 \mathrm{H}), 7.37$ (d, $J=$ $7.8 \mathrm{~Hz}, 1 \mathrm{H}), 7.24(\mathrm{td}, J=1.2, J=7.5 \mathrm{~Hz}, 1 \mathrm{H}), 7.16(\mathrm{~m}, 1 \mathrm{H}), 2.62(\mathrm{~s}$, $3 \mathrm{H}), 2.50(\mathrm{~s}, 3 \mathrm{H}) ;{ }^{13} \mathrm{C} \mathrm{NMR}\left(75 \mathrm{MHz}, \mathrm{CDCl}_{3}\right) \delta 197.6,137.9,136.3,135.3,132.6,129.9,128.9$, 127.3, 123.1, 119.8, 119.3, 110.9, 110.7, 26.6, 9.9. IR (film, $\mathrm{cm}^{-1}$ ): 3365, 1668, 1602, 740. HRMS for $\mathrm{C}_{17} \mathrm{H}_{15} \mathrm{NO}\left(\mathrm{M}^{+}\right)$calcd (found): 249.1154 (249.1154).

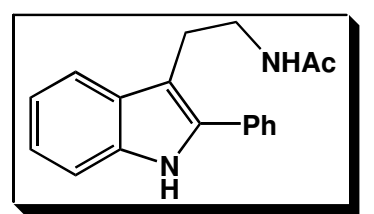

3-(2-Acetylamidoethyl)-2-phenylindole (Table 3, entry 4). Procedure B, $41 \%\left(\mathrm{SiO}_{2}, 1: 1\right.$ gradient to 3:1 EtOAc:hexanes): ${ }^{1} \mathrm{H}$ NMR $(300 \mathrm{MHz}$, DMSO- $\left.d_{6}\right) \delta 8.57(\mathrm{~s}, 1 \mathrm{H}), 7.63(\mathrm{~d}, J=7.8 \mathrm{~Hz}, 1 \mathrm{H}), 7.55-7.58(\mathrm{~m}, 2 \mathrm{H})$, 7.22-7.47 (m, $4 \mathrm{H}), 7.21(\mathrm{dt}, J=1.2,7.8 \mathrm{~Hz}, 1 \mathrm{H}), 7.14(\mathrm{dt}, J=1.2,8.1 \mathrm{~Hz}, 1$ H), 5.56 (bs, $1 \mathrm{H}), 3.53(\mathrm{q}, J=6.6 \mathrm{~Hz}, 2 \mathrm{H}), 3.11(\mathrm{t}, J=6.3 \mathrm{~Hz}, 2 \mathrm{H}), 1.73(\mathrm{~s}$, $3 \mathrm{H}) ;{ }^{13} \mathrm{C}$ NMR $\left(75 \mathrm{MHz}\right.$, DMSO- $\left.d_{6}\right) \delta 170.1,135.9,135.3,132.9,128.9,128.0,127.8,122.4$, 
$119.8,118.8,111.0,109.7,40.1,24.4,23.1$. One carbon peak in aromatic region is missing in our assignment, presumably due to overlap. IR (film, $\mathrm{cm}^{-1}$ ): $3277,1654,1527,1458,742,698$. HRMS for $\mathrm{C}_{18} \mathrm{H}_{18} \mathrm{~N}_{2} \mathrm{O}\left(\mathrm{M}^{+}\right)$calcd (found): 278.1419 (278.1412).

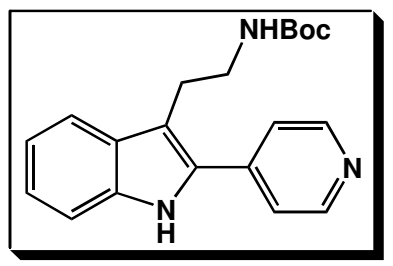

3-(2-Boc-aminoethyl)-2-(4-pyridyl)indole (Table 3, entry 5). Procedure B, $25 \%\left(\mathrm{SiO}_{2}, 2: 1\right.$ EtOAc:hexanes gradient to EtOAc/5\% MeOH): ${ }^{1} \mathrm{H}$ NMR $\left(300 \mathrm{MHz}, \mathrm{CDCl}_{3}\right) \delta 8.84(\mathrm{~s}, 1 \mathrm{H}), 6.63(\mathrm{~d}, J=6.0 \mathrm{~Hz}, 2 \mathrm{H}), 7.67(\mathrm{~d}, J=$ $7.8 \mathrm{~Hz}, 1 \mathrm{H}), 7.42-7.49(\mathrm{~m}, 2 \mathrm{H}), 7.41(\mathrm{~d}, J=8.1 \mathrm{~Hz}, 1 \mathrm{H}), 7.27$ (dd, $J=$ $1.2,4.8 \mathrm{~Hz}, 1 \mathrm{H}), 7.16(\mathrm{dd}, J=1.2,8.1 \mathrm{~Hz}, 1 \mathrm{H}), 4.72(\mathrm{bs}, 1 \mathrm{H}), 3.44-3.51$ $(\mathrm{m}, 2 \mathrm{H}), 3.15(\mathrm{t}, J=6.9 \mathrm{~Hz}, 2 \mathrm{H}), 1.41(\mathrm{~s}, 9 \mathrm{H}) ;{ }^{13} \mathrm{C} \mathrm{NMR}\left(75 \mathrm{MHz}, \mathrm{CDCl}_{3}\right) \delta 155.9,150.1,140.5$, 136.5, 131.9, 129.0, 123.6, 121.9, 120.2, 119.6, 112.9, 111.2, 79.3, 41.0, 28.4, 25.5. IR (film, $\mathrm{cm}^{-1}$ ): 2976, 1670, 1603, 1165, 735. HRMS for $\mathrm{C}_{20} \mathrm{H}_{23} \mathrm{~N}_{3} \mathrm{O}_{2}$ (M $\mathrm{M}^{+}$) calcd (found): 338.1869 (338.1869).

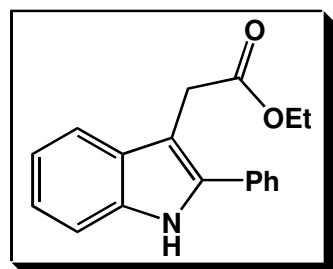

3-(Ethoxycarbonylmethyl)-2-phenylindole (Table 3, entry 6). Procedure $\mathrm{B}$, 46\% $\left(\mathrm{SiO}_{2}, 5: 1\right.$ hexanes:EtOAc): ${ }^{1} \mathrm{H}$ NMR $\left(400 \mathrm{MHz}, \mathrm{CDCl}_{3}\right) \delta 8.13(\mathrm{~s}, 1 \mathrm{H})$, $7.66(\mathrm{~m}, 3 \mathrm{H}), 7.47(\mathrm{~m}, 2 \mathrm{H}), 7.40-7.35(\mathrm{~m}, 2 \mathrm{H}), 7.21(\mathrm{dt}, J=1.2,7.6 \mathrm{~Hz}, 1$ $\mathrm{H}), 7.15(\mathrm{~m}, 1 \mathrm{H}), 4.16$ (quat, $J=7.1 \mathrm{~Hz}, 2 \mathrm{H}), 3.83(\mathrm{~s}, 2 \mathrm{H}), 1.25$ (t, $J=7.1$ $\mathrm{Hz}, 3 \mathrm{H}) ;{ }^{13} \mathrm{C}$ NMR $\left(100 \mathrm{MHz}, \mathrm{CDCl}_{3}\right) \delta 171.9,136.0,135.5,132.2,128.9$, $128.7,128.1,127.9,122.4,119.9,119.2,110.7,105.7,60.9,31.3,14.4$. IR (film, $\mathrm{cm}^{-1}$ ): 2359, 1718, 1457, 743, 700. HRMS for $\mathrm{C}_{18} \mathrm{H}_{17} \mathrm{NO}_{2}\left(\mathrm{M}^{+}\right)$calcd (found): 279.1259 (279.1262).

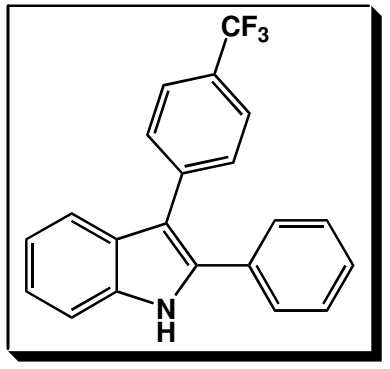

3-(4-Trifluoromethylphenyl)-2-phenylindole (Table 3, entry 7). Procedure $\mathrm{B}, 46 \%\left(\mathrm{SiO}_{2}\right.$, gravity column, 10:1 hexanes:EtOAc): ${ }^{1} \mathrm{H}$ NMR $\left(300 \mathrm{MHz}, \mathrm{CDCl}_{3}\right) \delta 8.25(\mathrm{~s}, 1 \mathrm{H}), 7.66(\mathrm{~d}, J=7.9 \mathrm{~Hz}, 1 \mathrm{H}), 7.60(\mathrm{~d}, J=$ $8.1 \mathrm{~Hz}, 2 \mathrm{H}), 7.53(\mathrm{~d}, J=8.1 \mathrm{~Hz}, 2 \mathrm{H}), 7.31-7.43(\mathrm{~m}, 6 \mathrm{H}), 7.26(\mathrm{~m}, 1 \mathrm{H})$, $7.17(\mathrm{~m}, 1 \mathrm{H}) ;{ }^{13} \mathrm{C}$ NMR $\left(75 \mathrm{MHz}, \mathrm{CDCl}_{3}\right) \delta 139.0,135.9,135.0,132.2$, $130.2,128.9,128.7,128.3,128.2\left(\mathrm{q}, J_{\mathrm{F}-\mathrm{C}}=32 \mathrm{~Hz}\right), 125.5\left(\mathrm{q}, J_{\mathrm{F}-\mathrm{C}}=270\right.$ $\mathrm{Hz}), 126.2,125.5\left(\mathrm{q}, J_{\mathrm{F}-\mathrm{C}}=11 \mathrm{~Hz}\right)$ (This long distance F-C coupling is only partially resolved), 123.0, 120.8, 119.3, 113.5, 111.1; ${ }^{19} \mathrm{~F} \mathrm{NMR}\left(282 \mathrm{Mhz}, \mathrm{CDCl}_{3}\right) \delta-62.2$. IR (film, $\mathrm{cm}^{-1}$ ): 2360, 1324, 1066. HRMS for $\mathrm{C}_{21} \mathrm{H}_{14} \mathrm{~F}_{3} \mathrm{~N}\left(\mathrm{MH}^{+}\right.$) calcd (found): 337.1078 (337.1092).

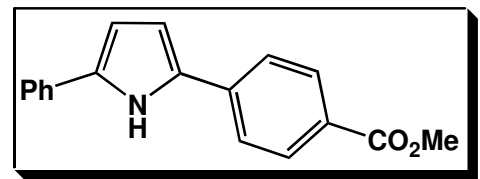

2-(4-Methoxycarbonylphenyl)-5-phenylpyrrole (Table 3, entry 8). Procedure $\mathrm{B}, 58 \%\left(\mathrm{SiO}_{2}\right.$, gravity column, 8:1 to 5:1 hexanes:EtOAc): ${ }^{1} \mathrm{H}$ NMR (400 MHz, DMSO- $\left.d_{6}\right) \delta 11.5$ (bs, $\left.1 \mathrm{H}\right), 7.91-7.98(\mathrm{~m}, 4 \mathrm{H})$, 7.80-7.83 (m, $2 \mathrm{H}), 7.40(\mathrm{t}, J=8.0 \mathrm{~Hz}, 2 \mathrm{H}), 7.22(\mathrm{t}, 7.6 \mathrm{~Hz}, 1 \mathrm{H})$, $6.80(\mathrm{dd}, J=2.8,3.6 \mathrm{~Hz}, 1 \mathrm{H}), 6.66(\mathrm{dd}, J=2.4,3.6 \mathrm{~Hz}, 1 \mathrm{H}), 3.86(\mathrm{~s}, 3 \mathrm{H}) ;{ }^{13} \mathrm{C} \mathrm{NMR}(100 \mathrm{MHz}$, DMSO- $\left.d_{6}\right) \delta 166.1,136.9,134.6,132.2,131.8,129.7,128.6,126.2,126.1,124.3,123.5,110.0$, 108.3, 51.9. IR (film, $\mathrm{cm}^{-1}$ ): 1707, 1294, 768, 747. HRMS for $\mathrm{C}_{18} \mathrm{H}_{15} \mathrm{NO}_{2}\left(\mathrm{M}^{+}\right)$calcd (found): 277.1103 (277.1103). 

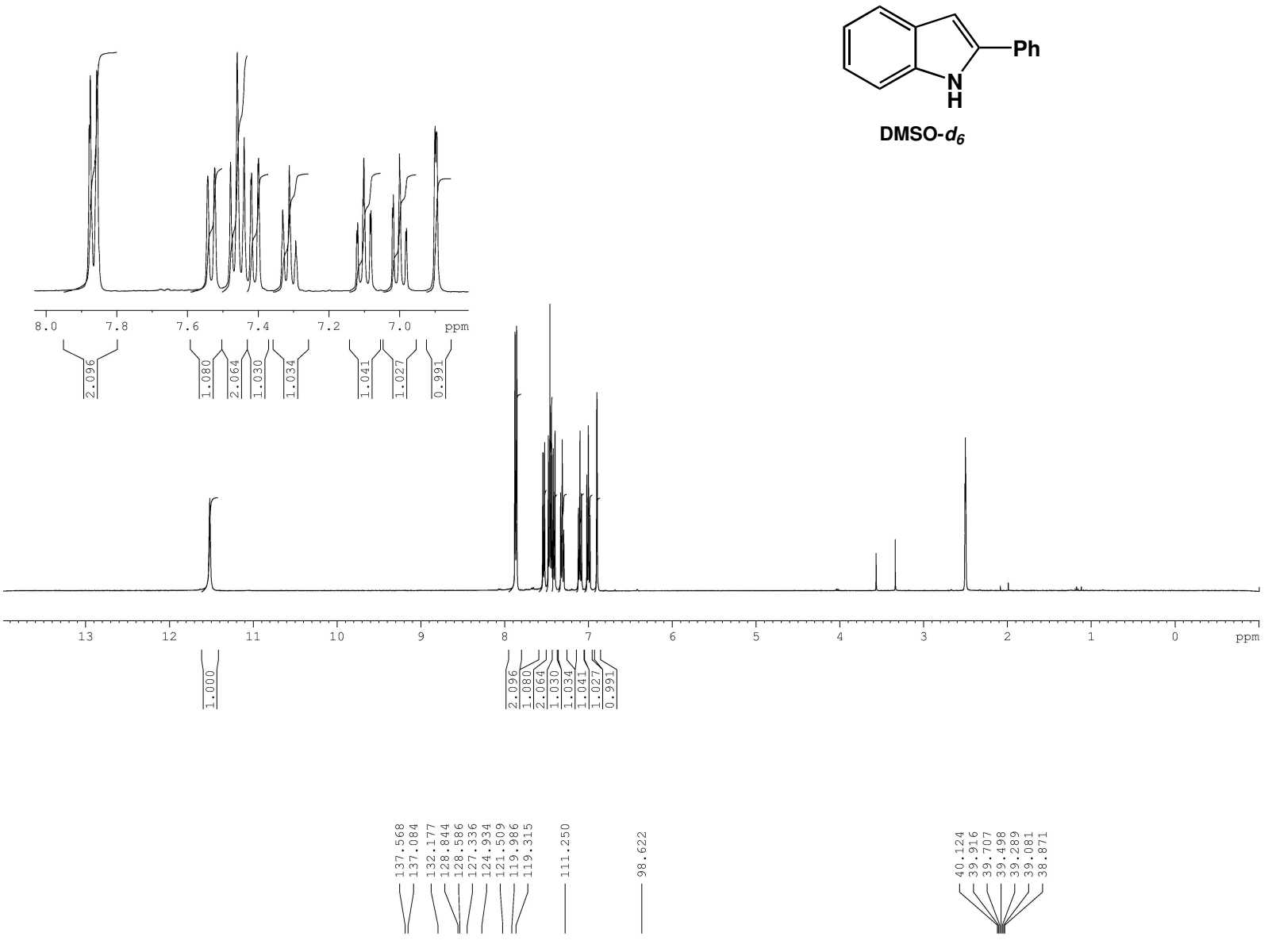

SW-III-197-2
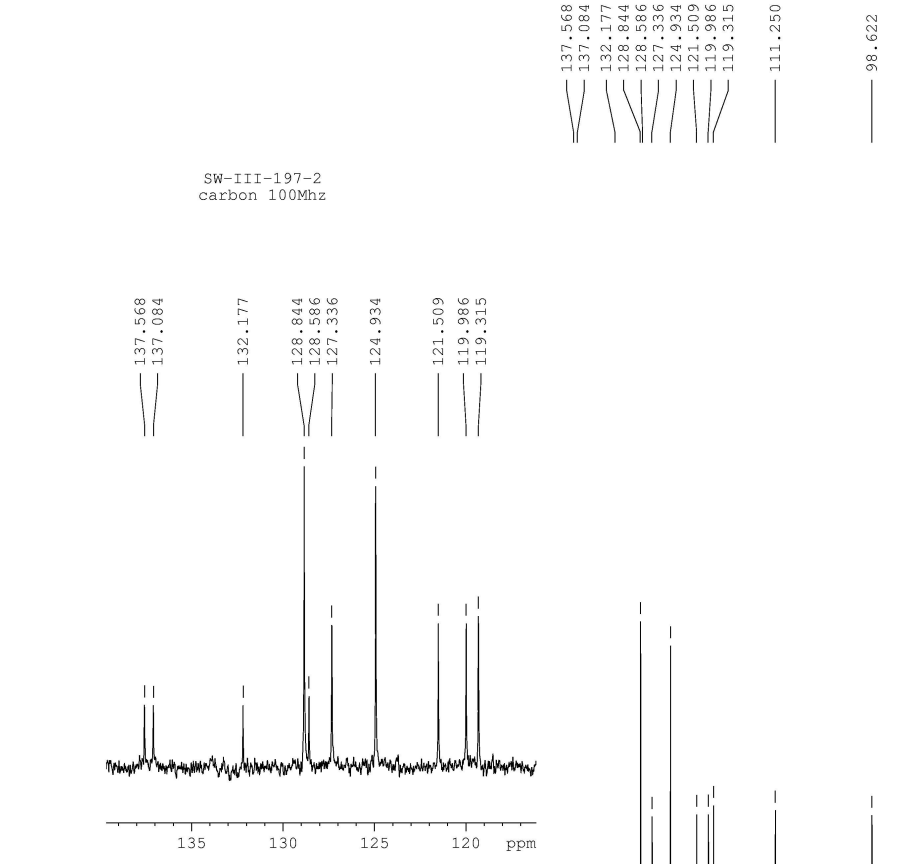

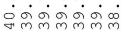
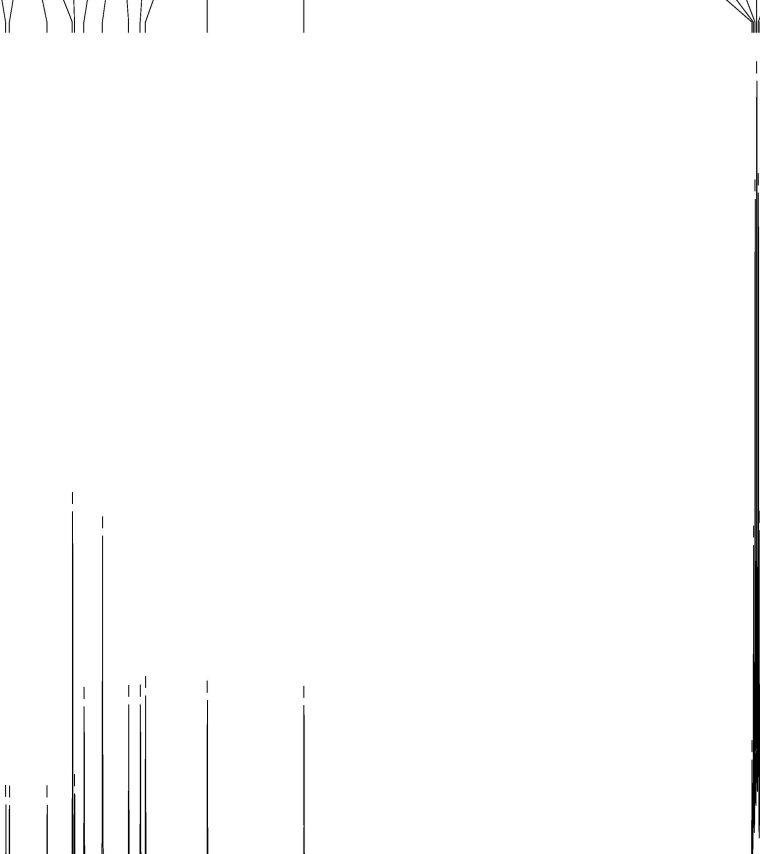


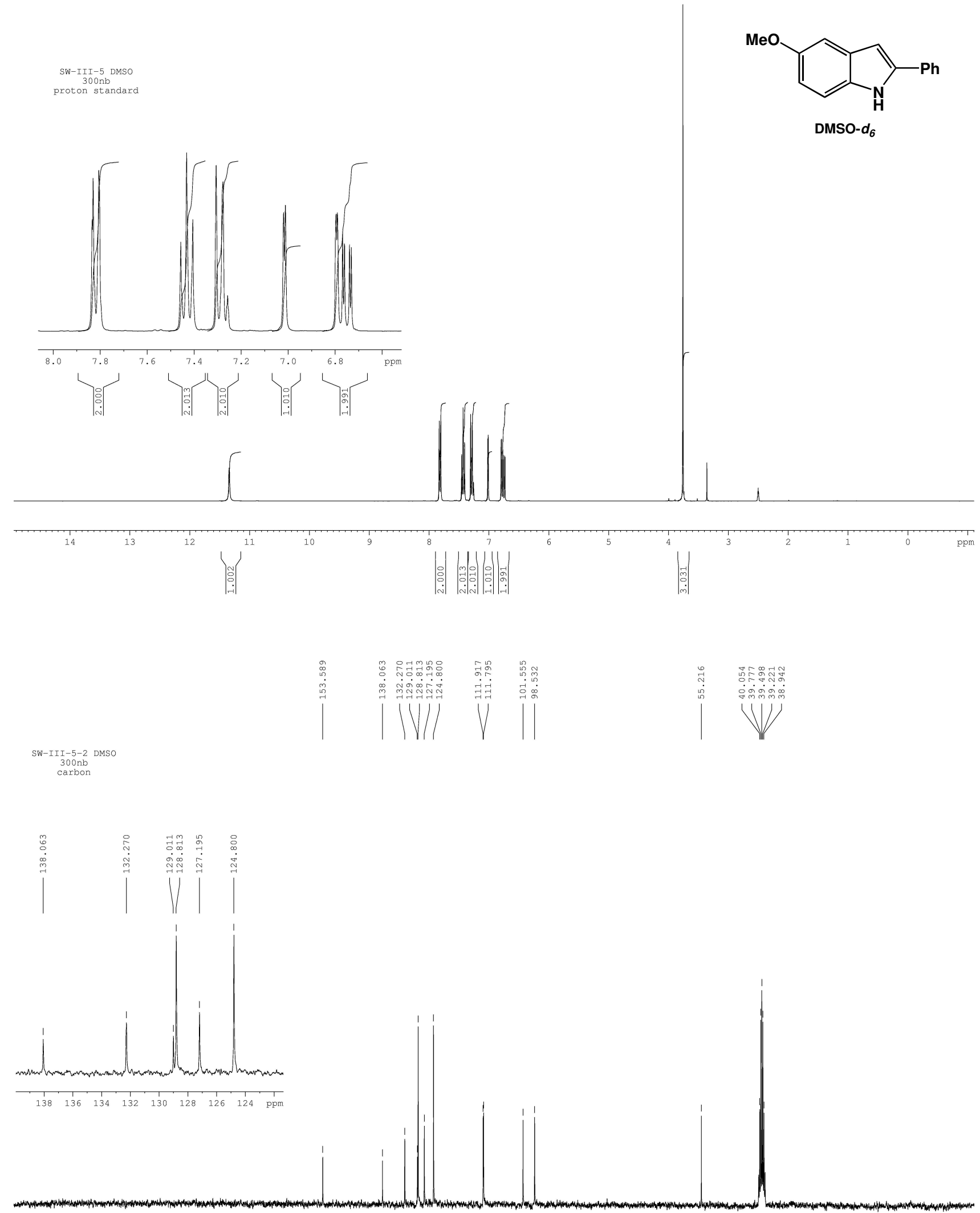

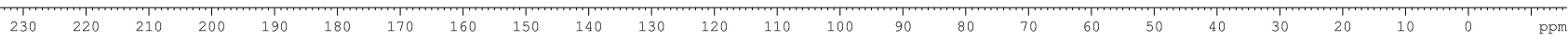




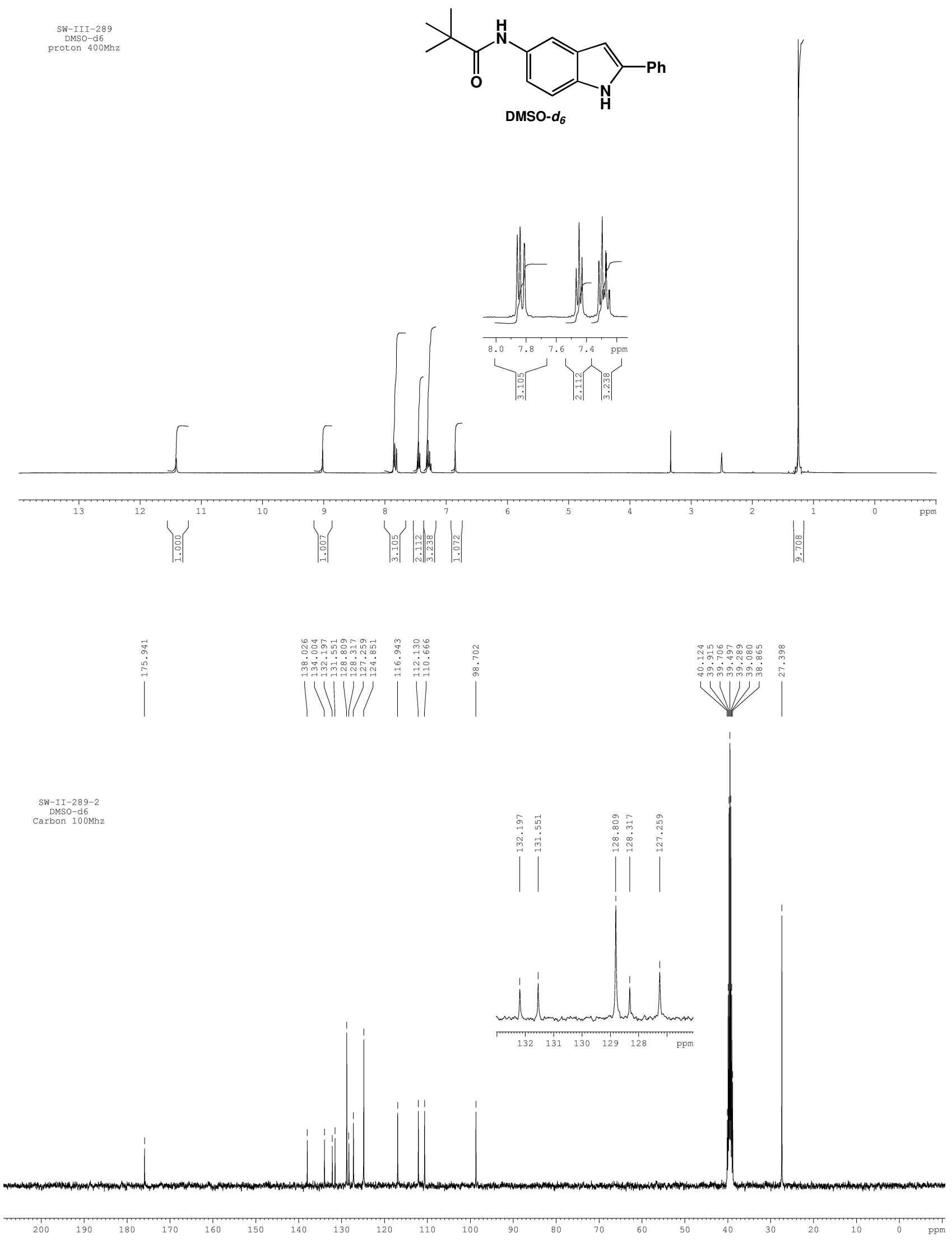


SW-III-158-1

$300 \mathrm{wb}$
proton

$\mathrm{MeO}$

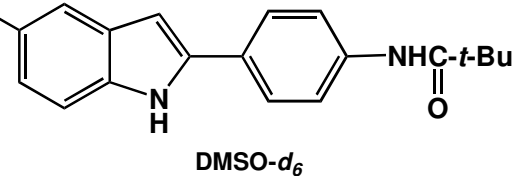

DMSO- $d_{6}$
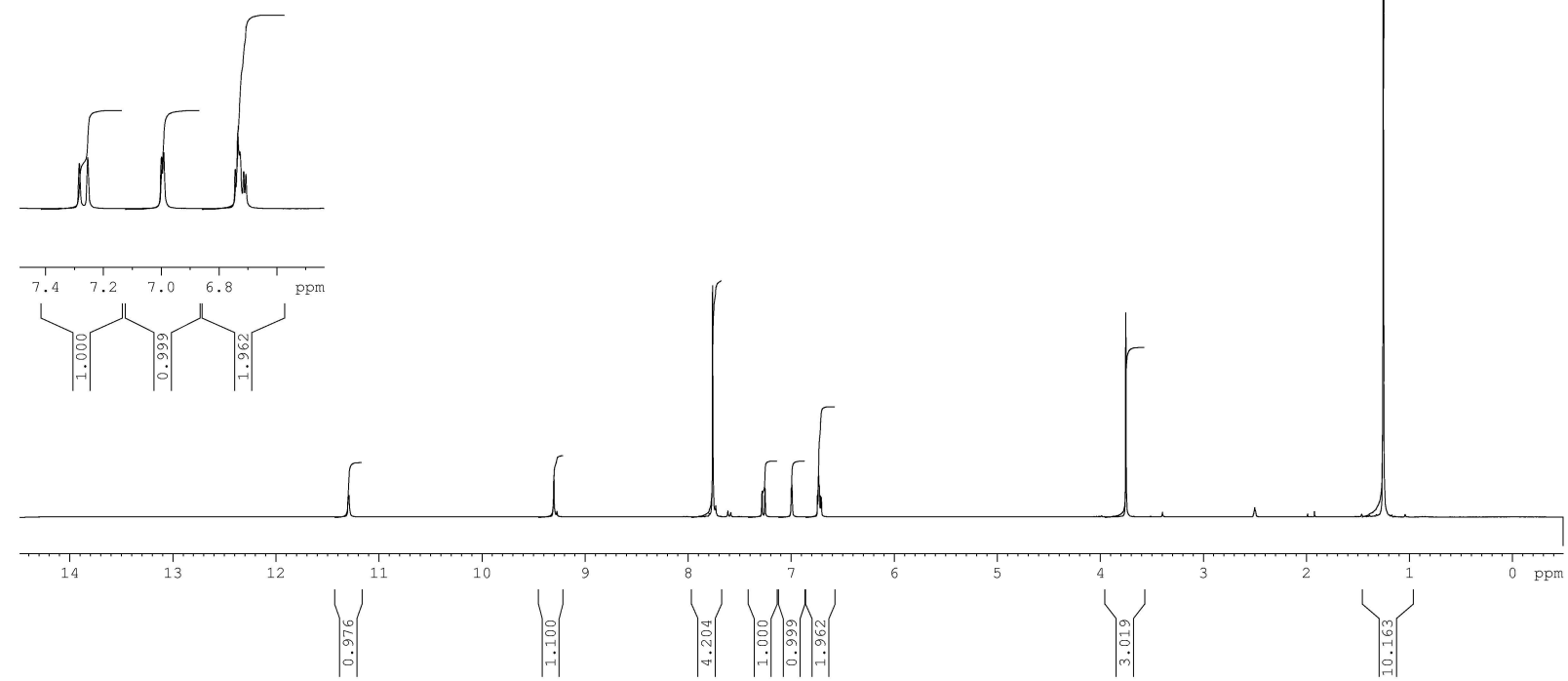

SW-III-158-2
300wb

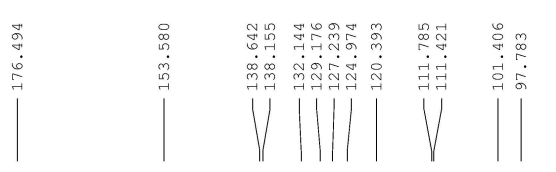

carbon NMR
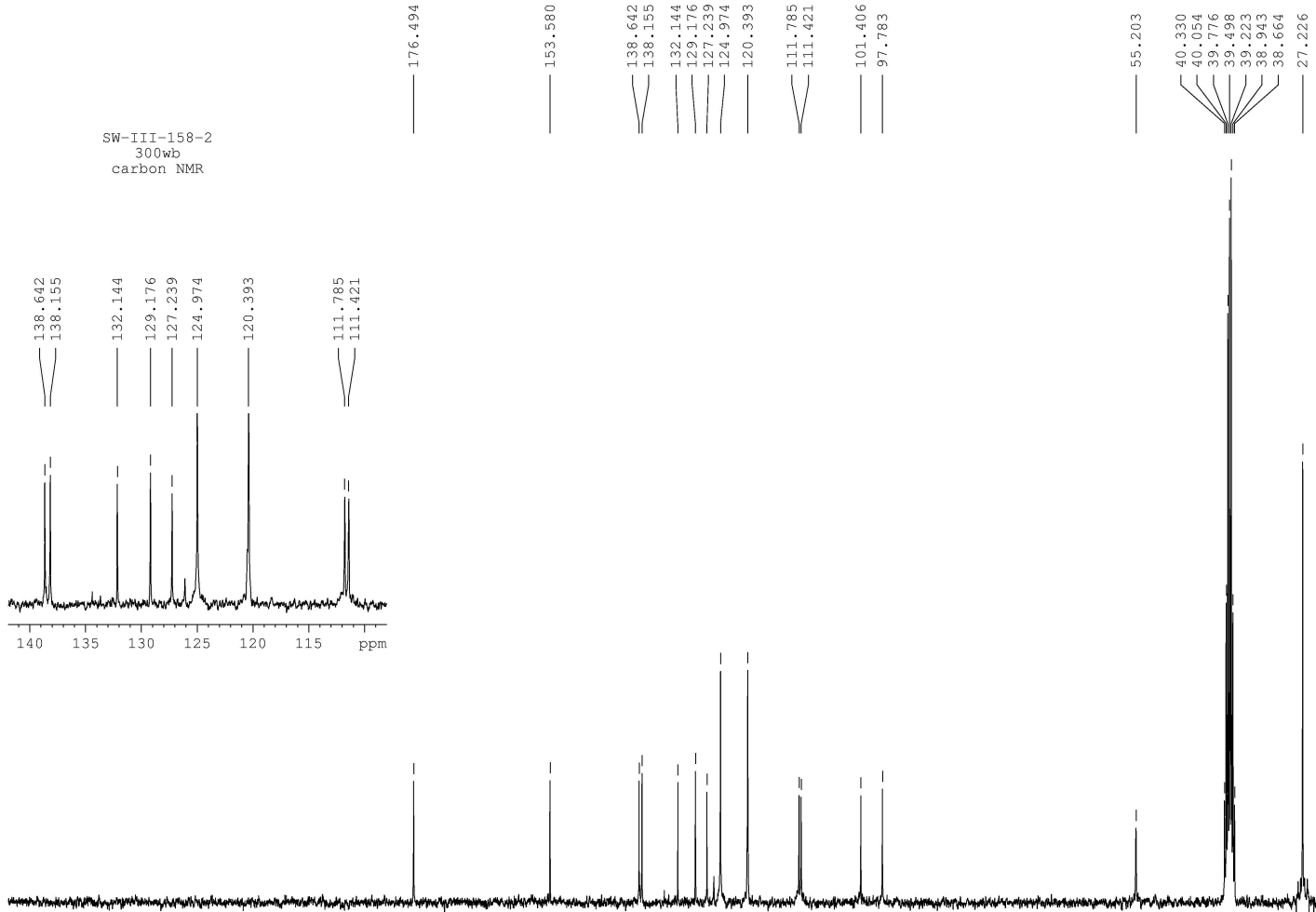
SW-III-156-1
300 wb

$300 w b$
proton

DMSO-d $d_{6}$
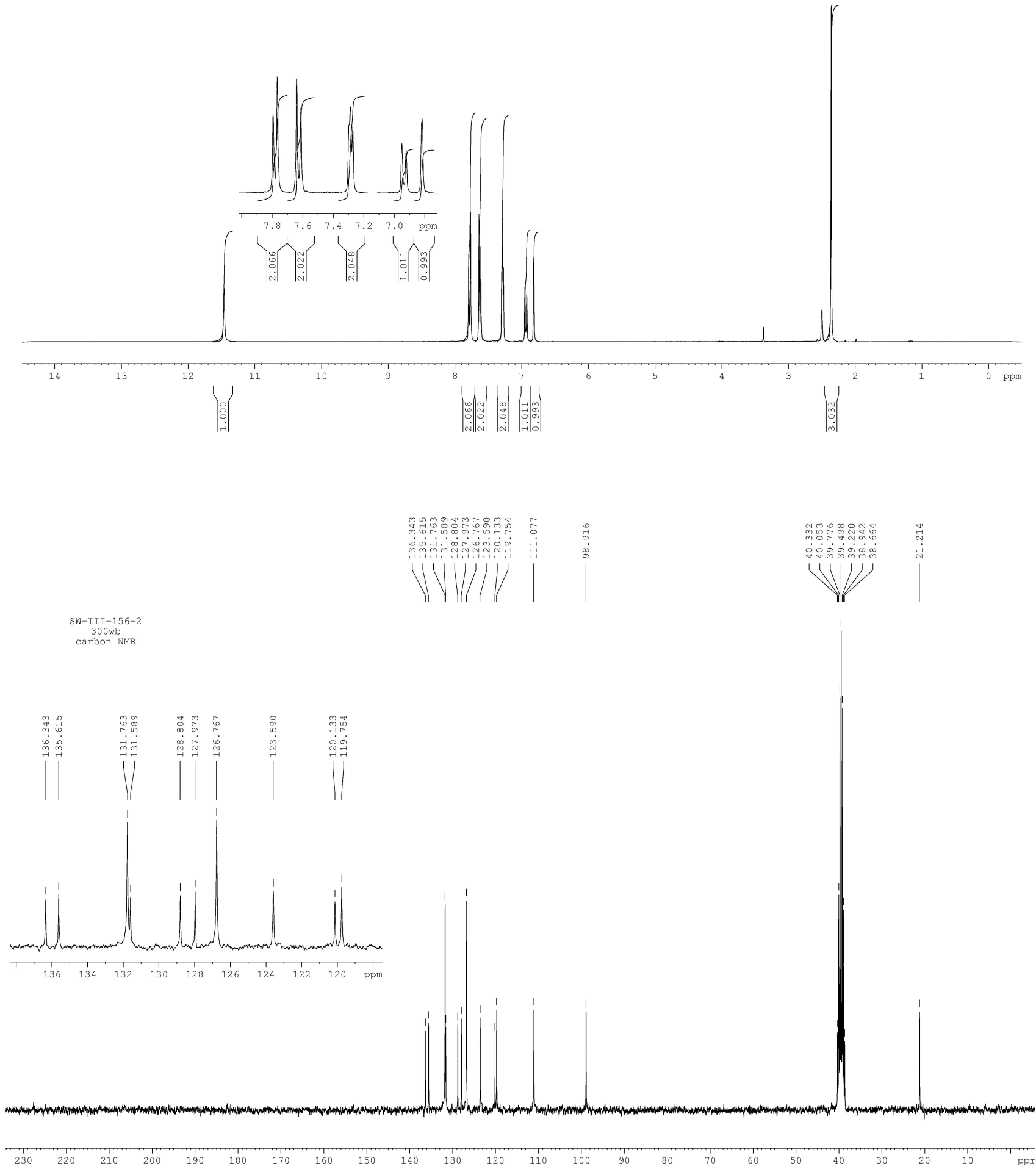
SW-III-194-1

$300 \mathrm{wb}$
proton
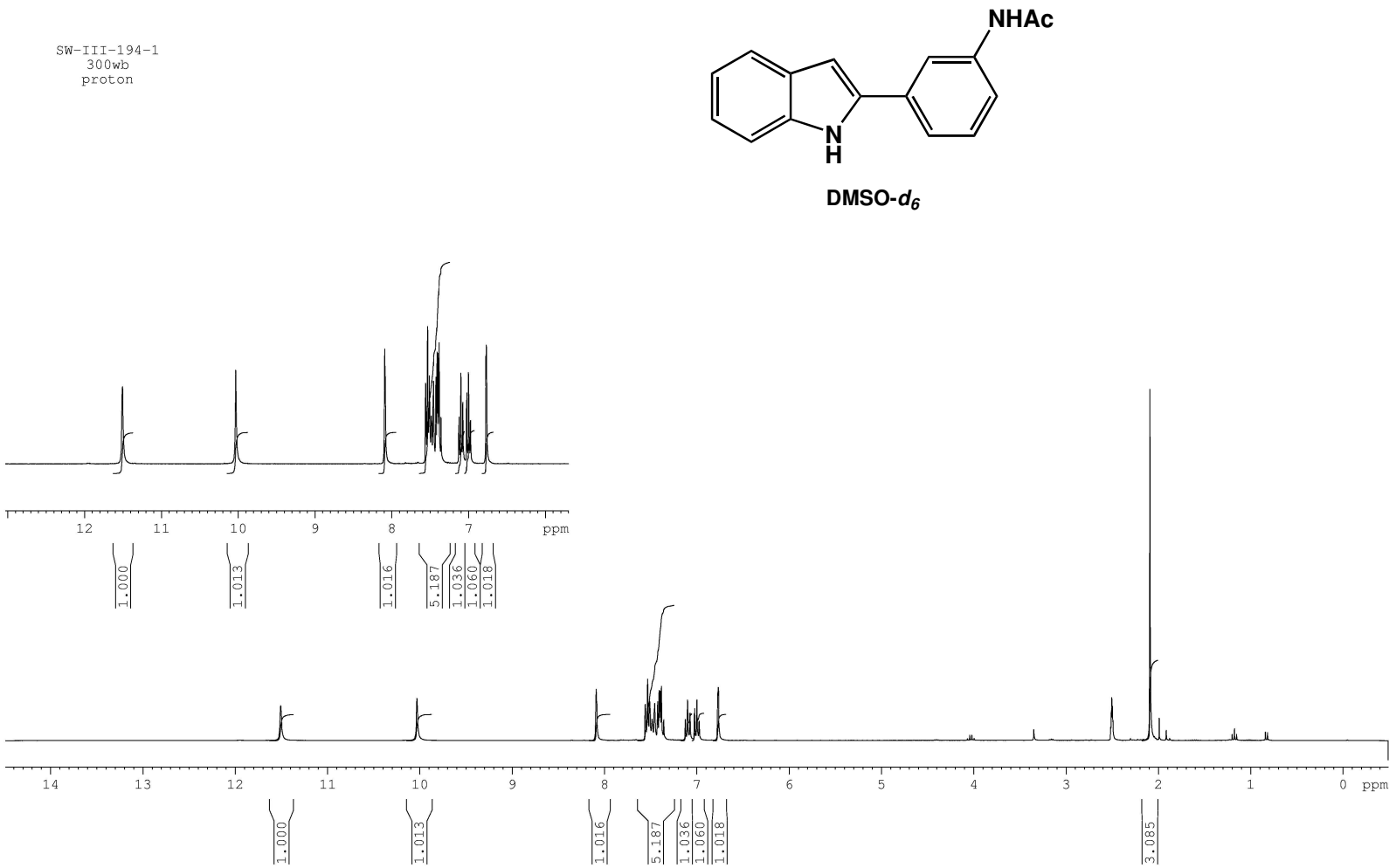

SW-III-194-2

carbon NMR

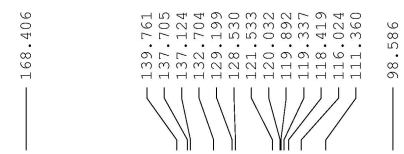

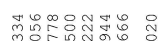

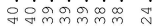

UV।

के

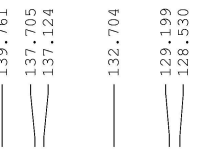

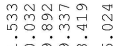

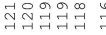

$|V|||$

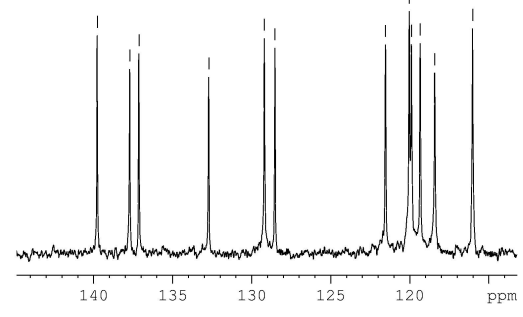

|
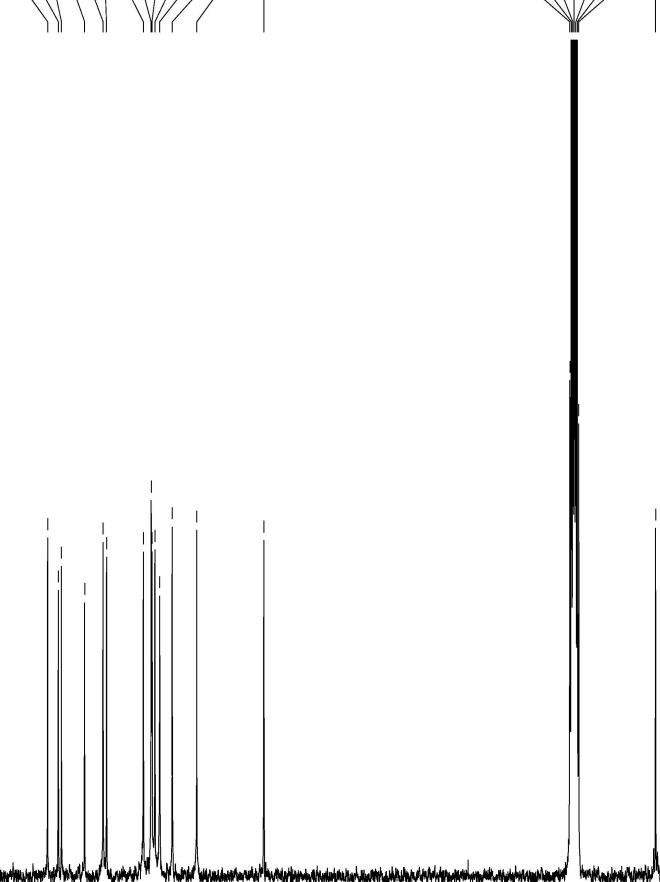

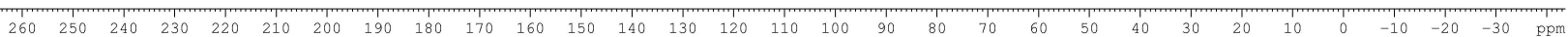


SW-III-162-1

proton
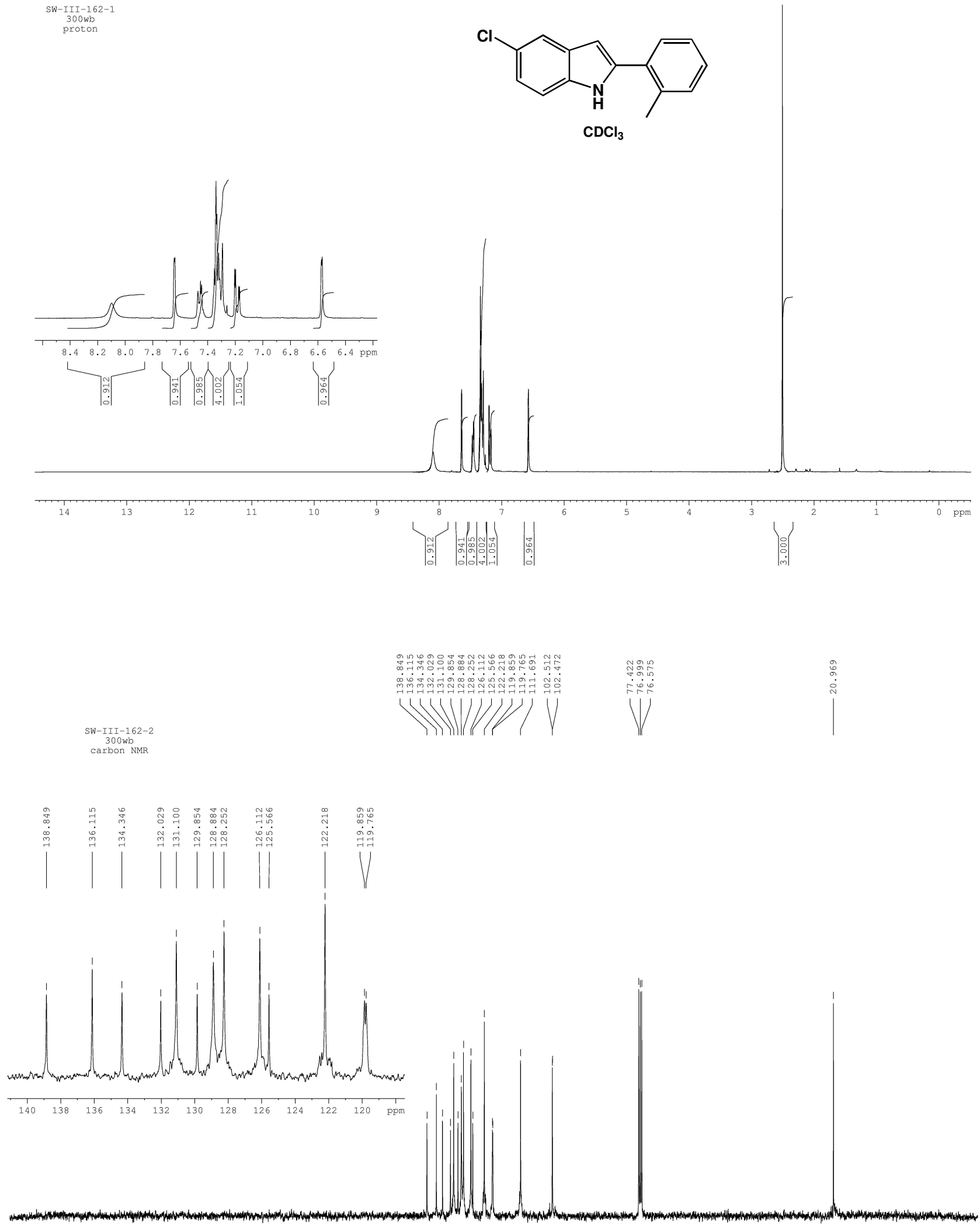

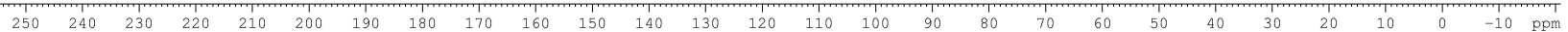



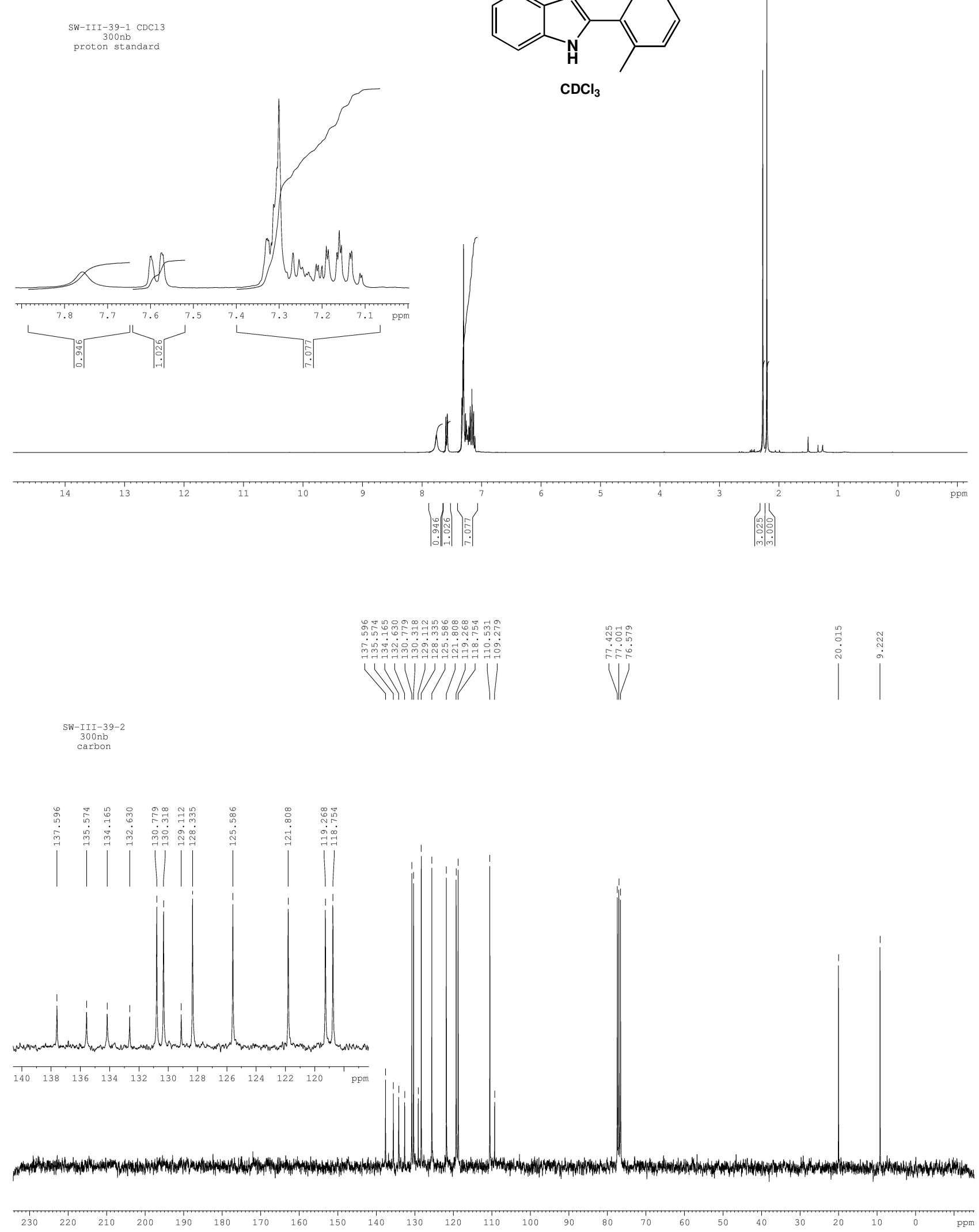

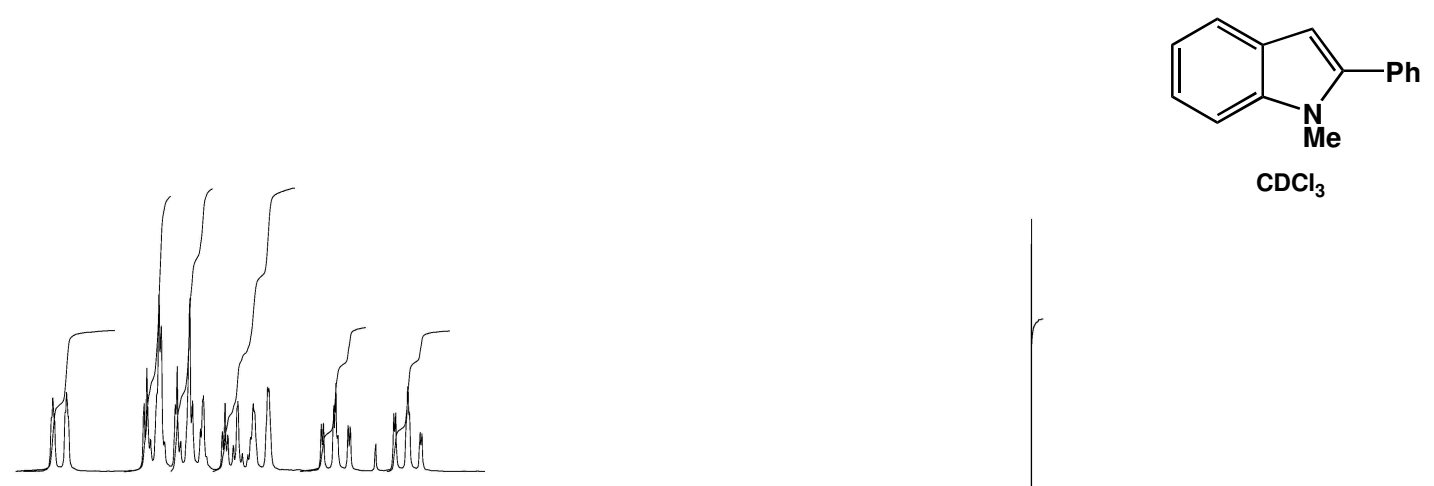

$\mathrm{CDCl}_{3}$
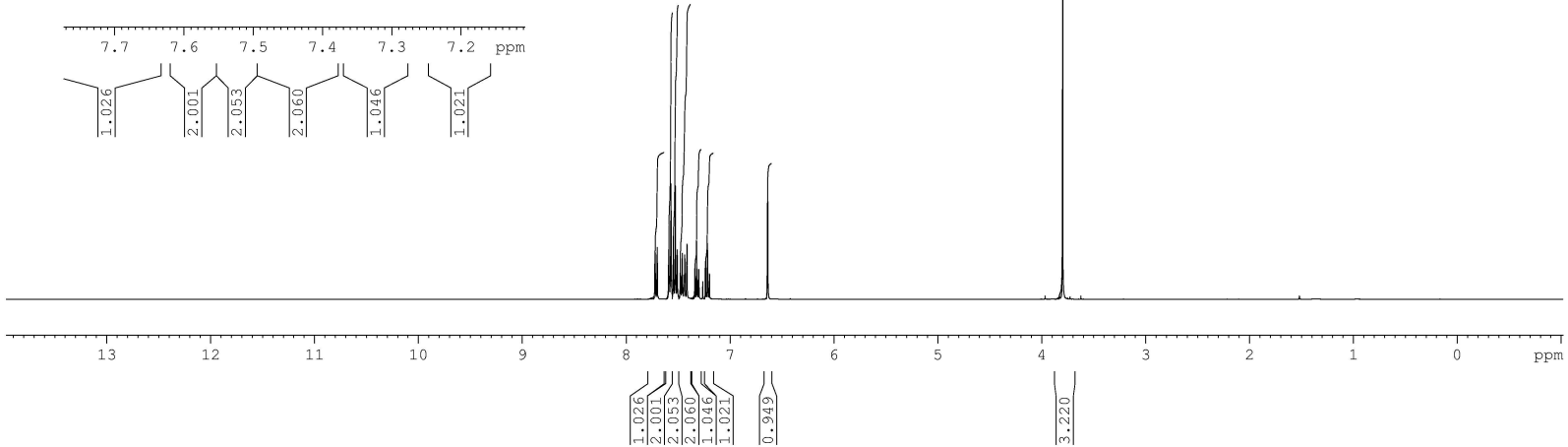

Carbon $100 \mathrm{MHz}$
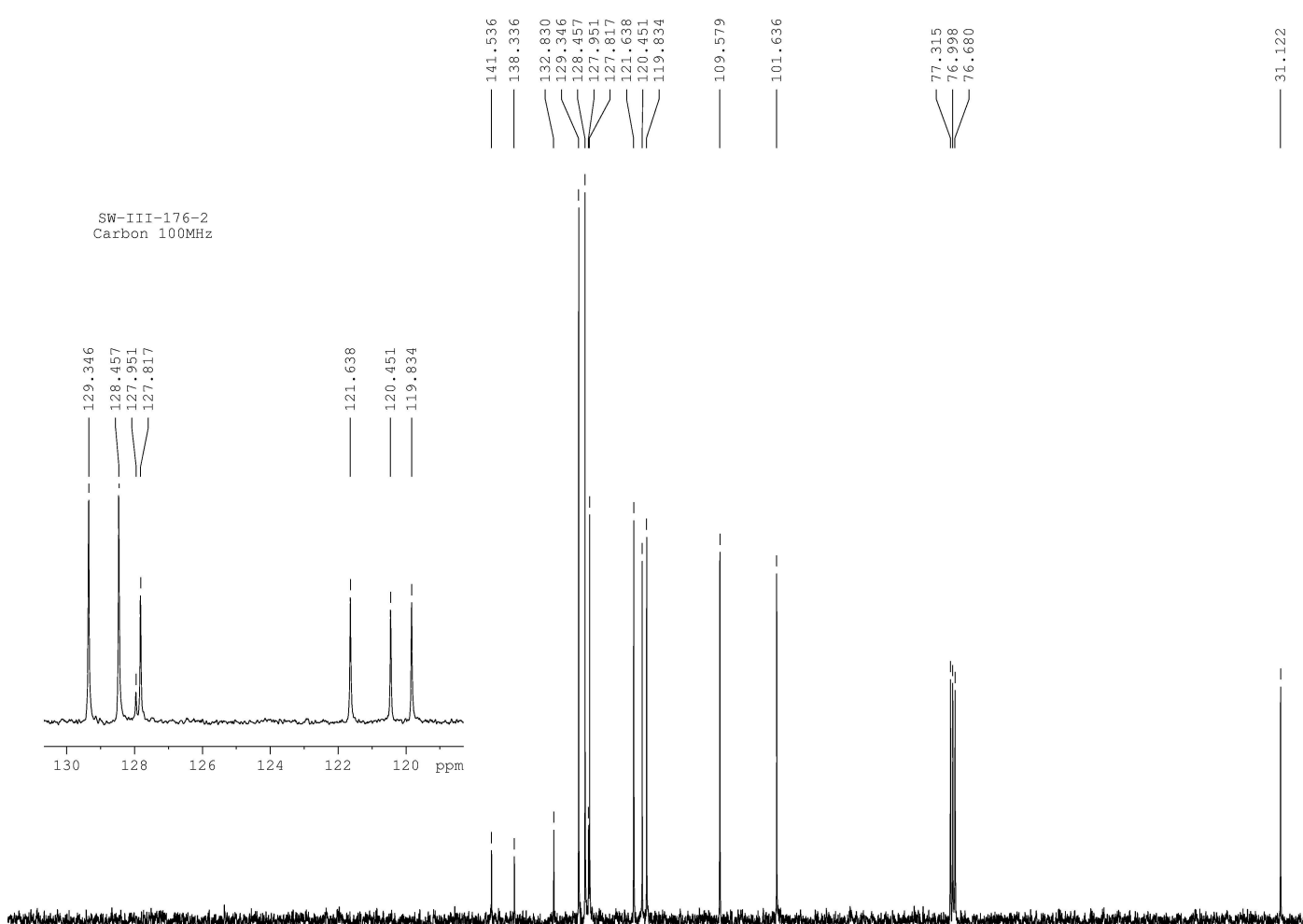

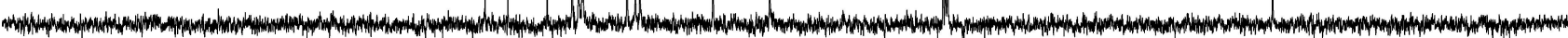




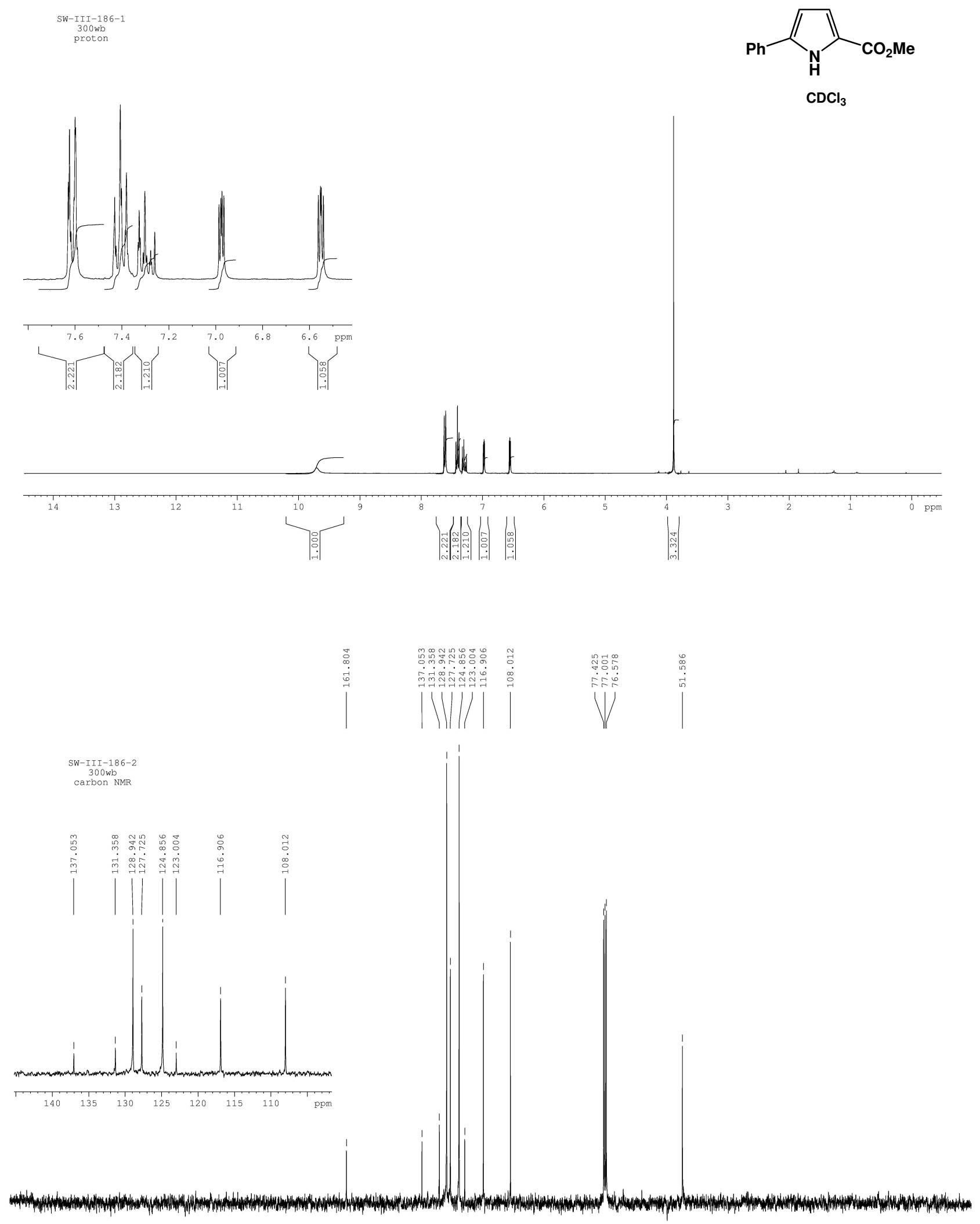

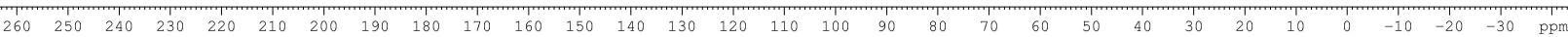


SW-DG-58 CDC13
RT $400 \mathrm{Mhz}$
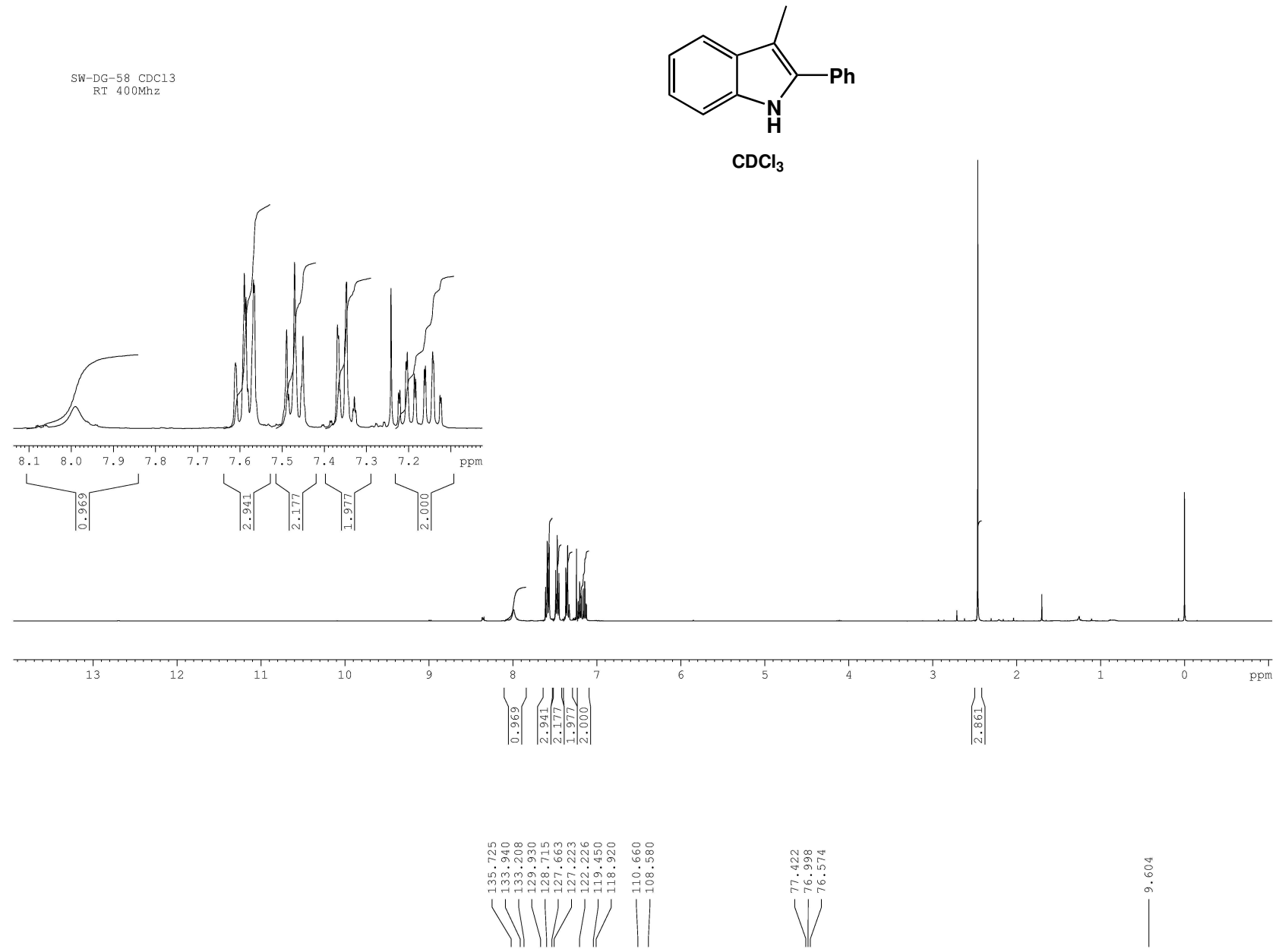

SW-III-58-2 300nb

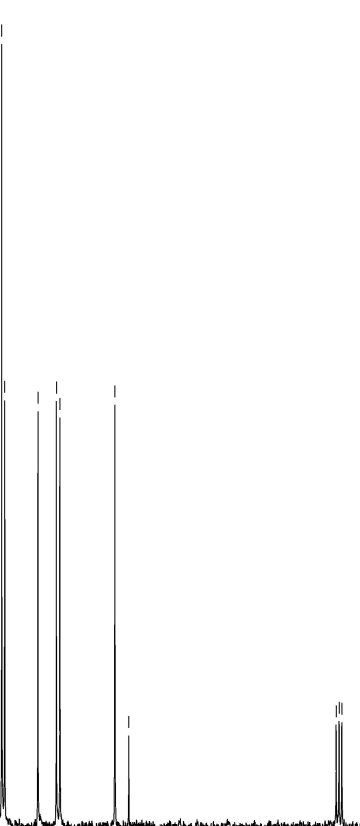

$\left.\right|_{136} ^{\mid}$ 
SW-III-184-1

300 wb
proton
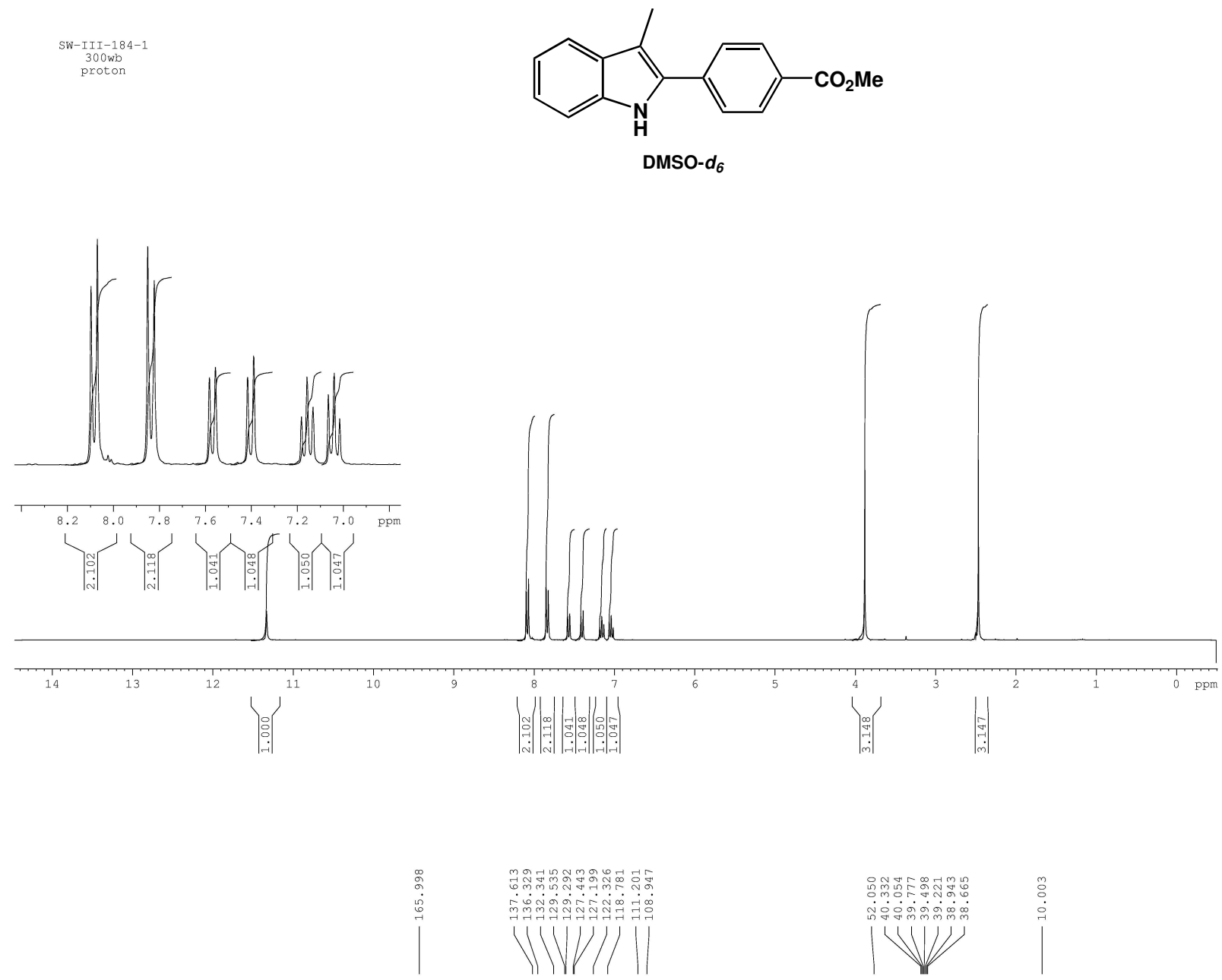

SW-III-184-2

carbon NMR

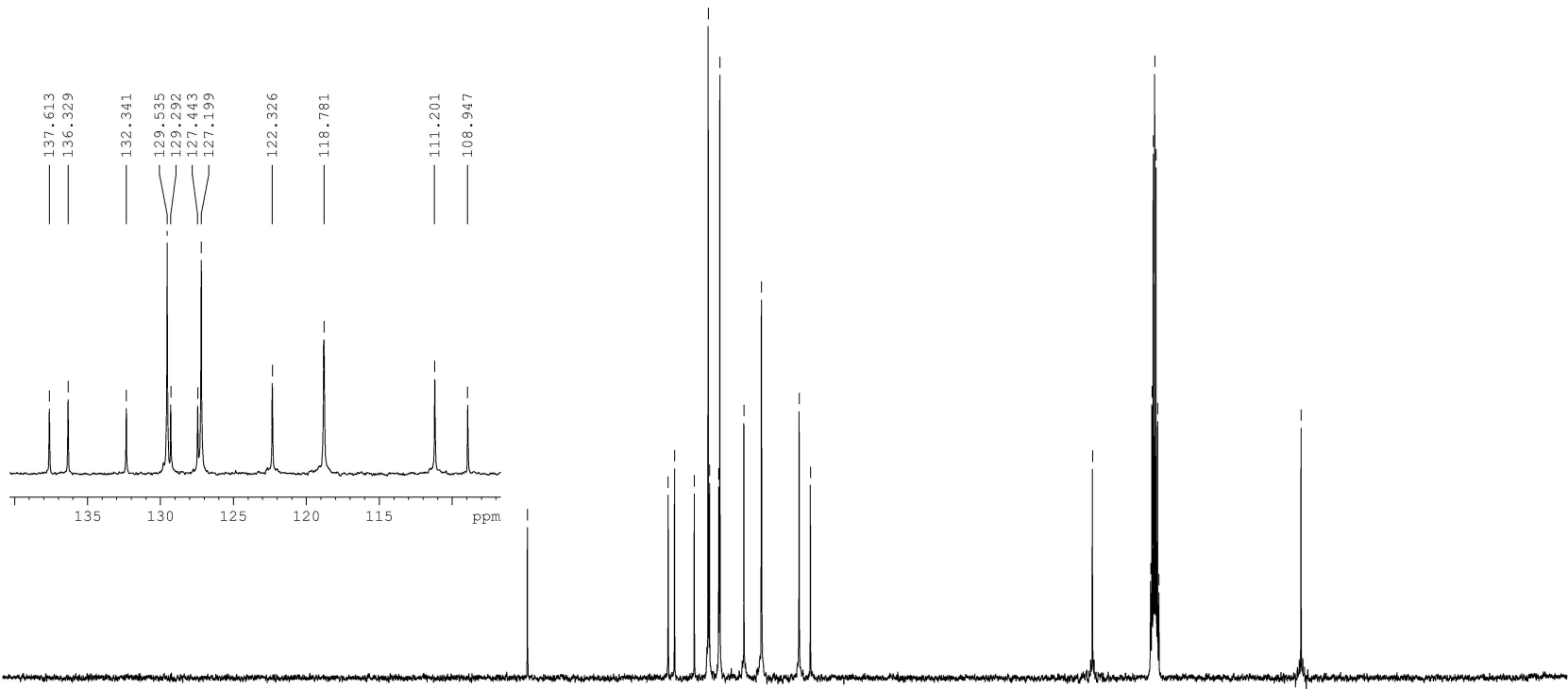

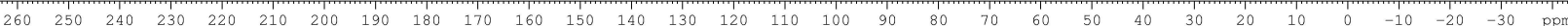


dg5 d
$300 \mathrm{MHz}$
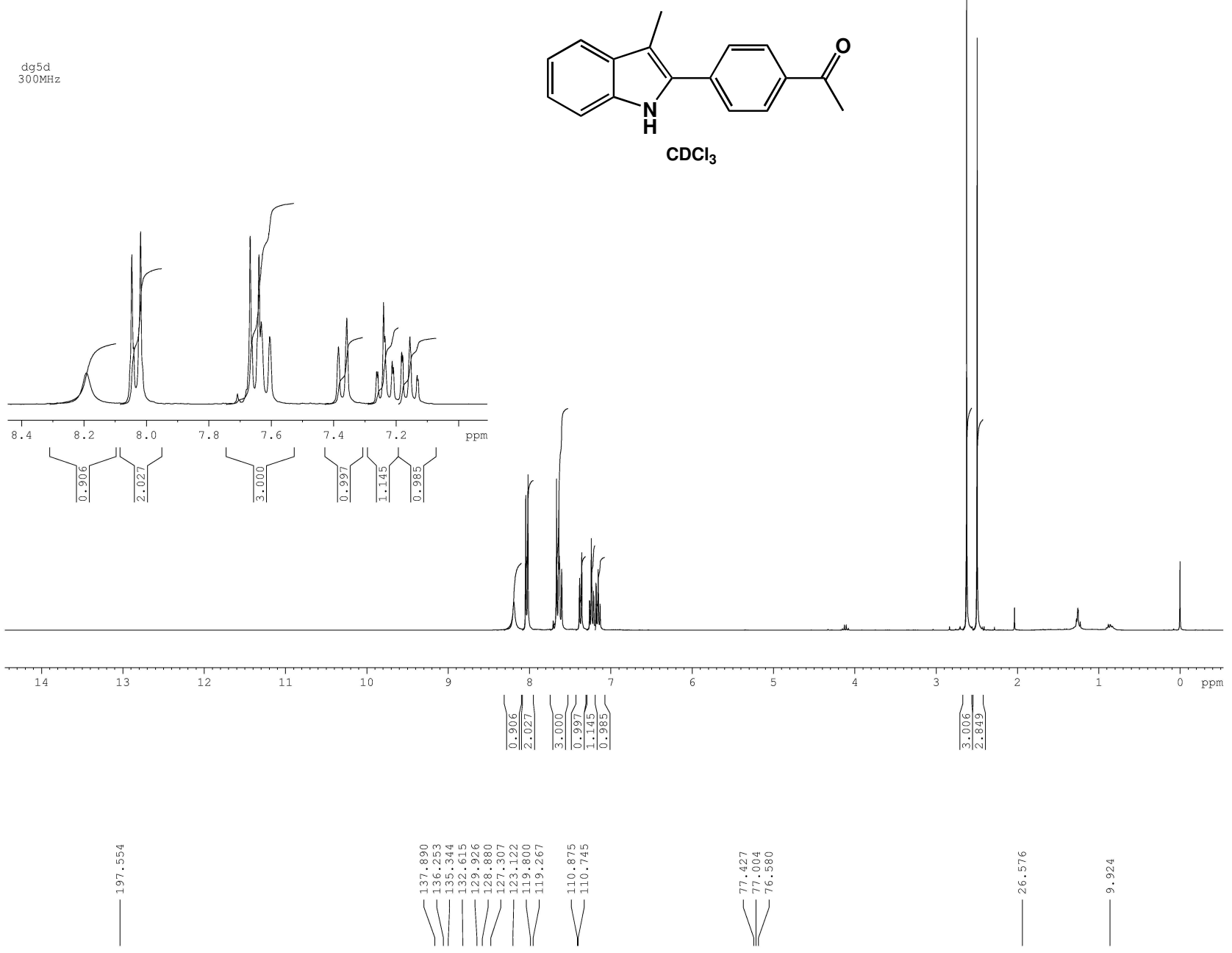

dg5d 300MHz CDCl3
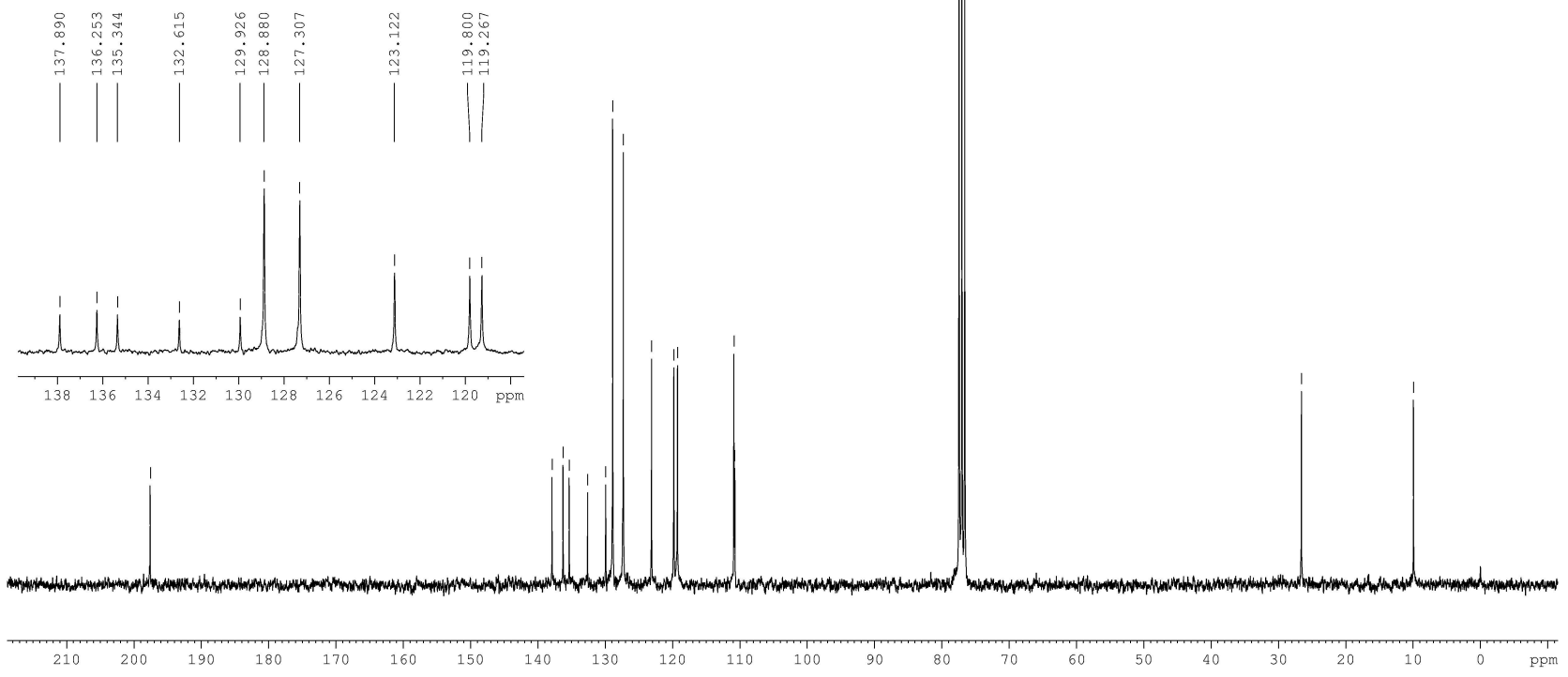


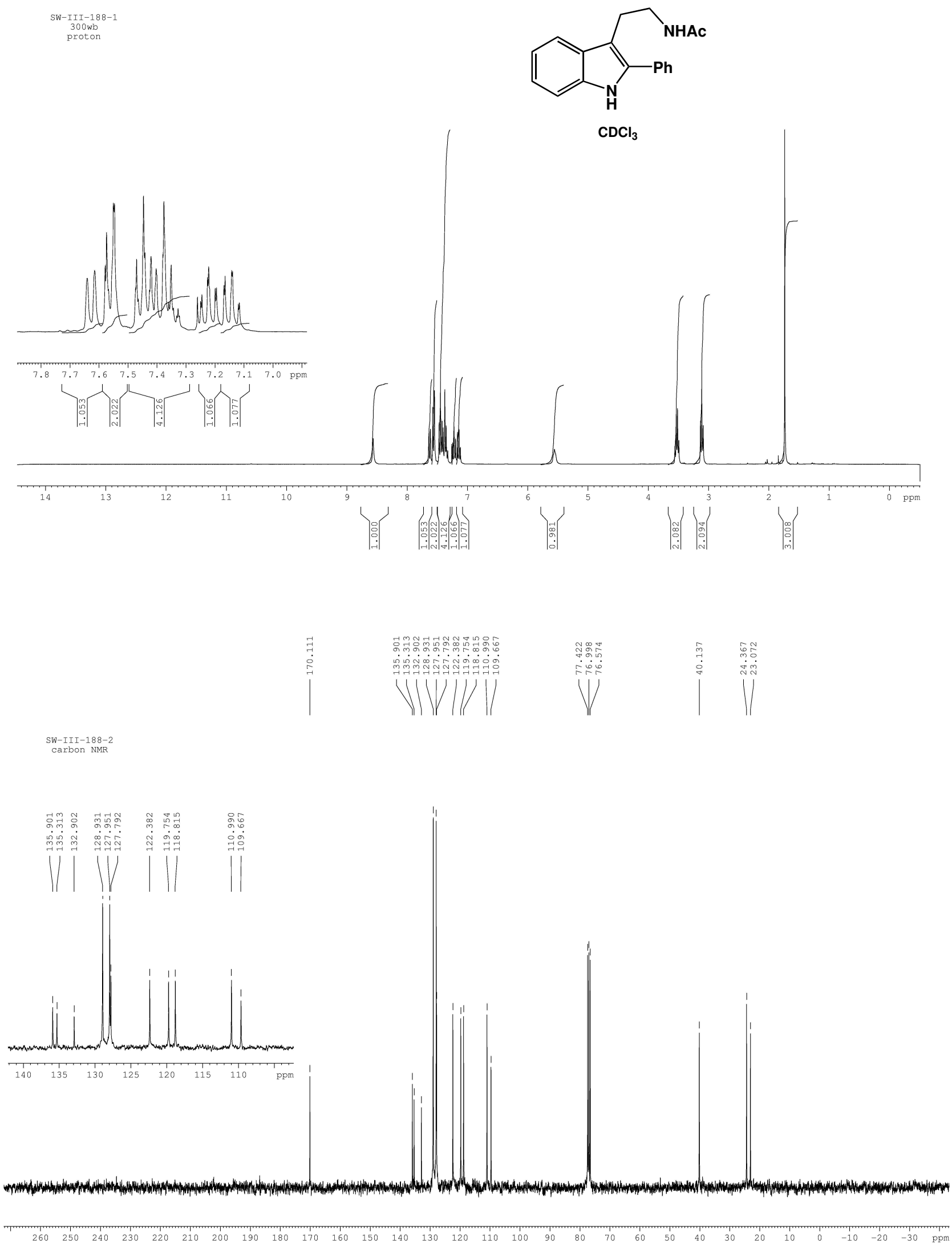




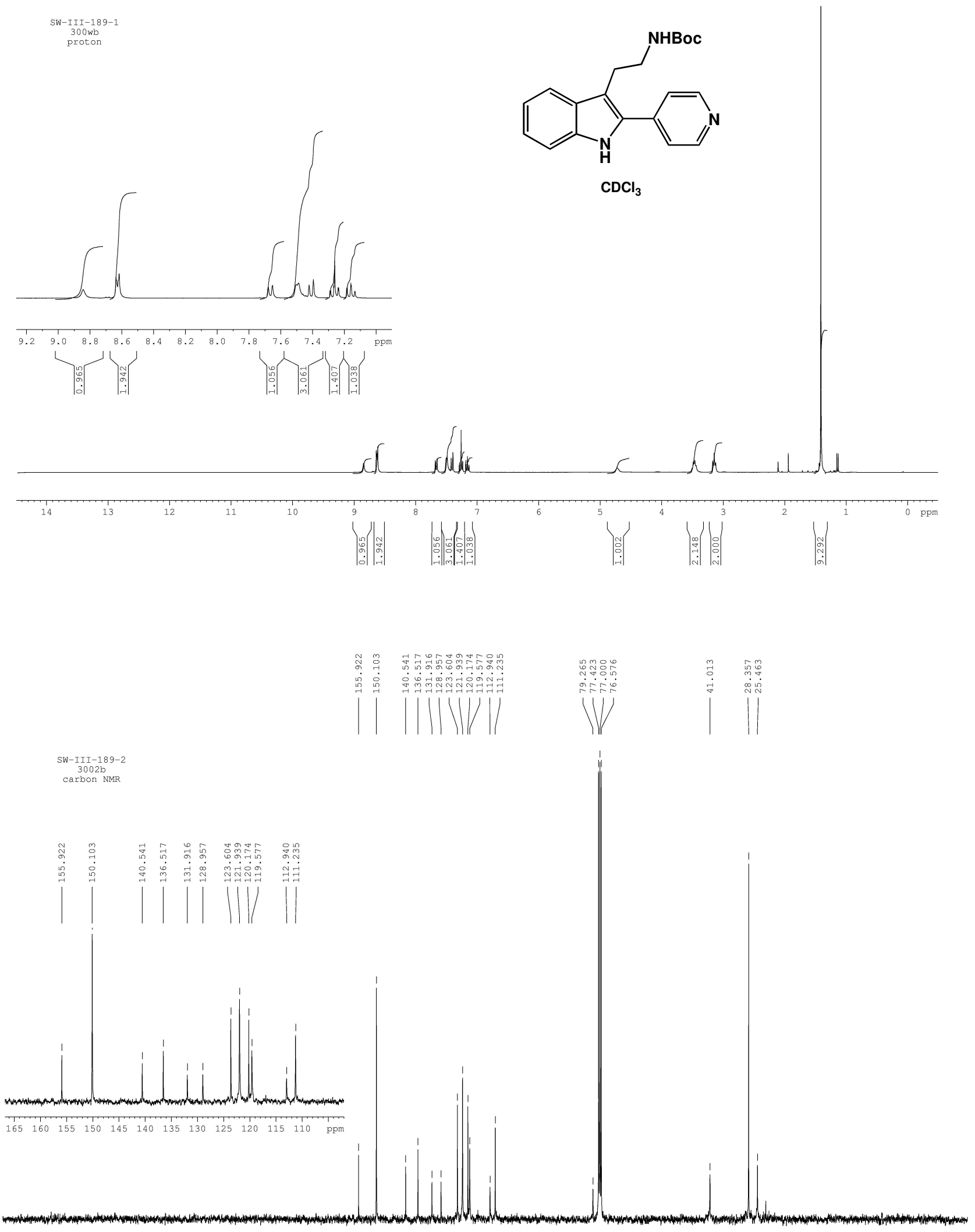

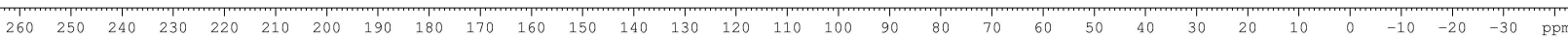




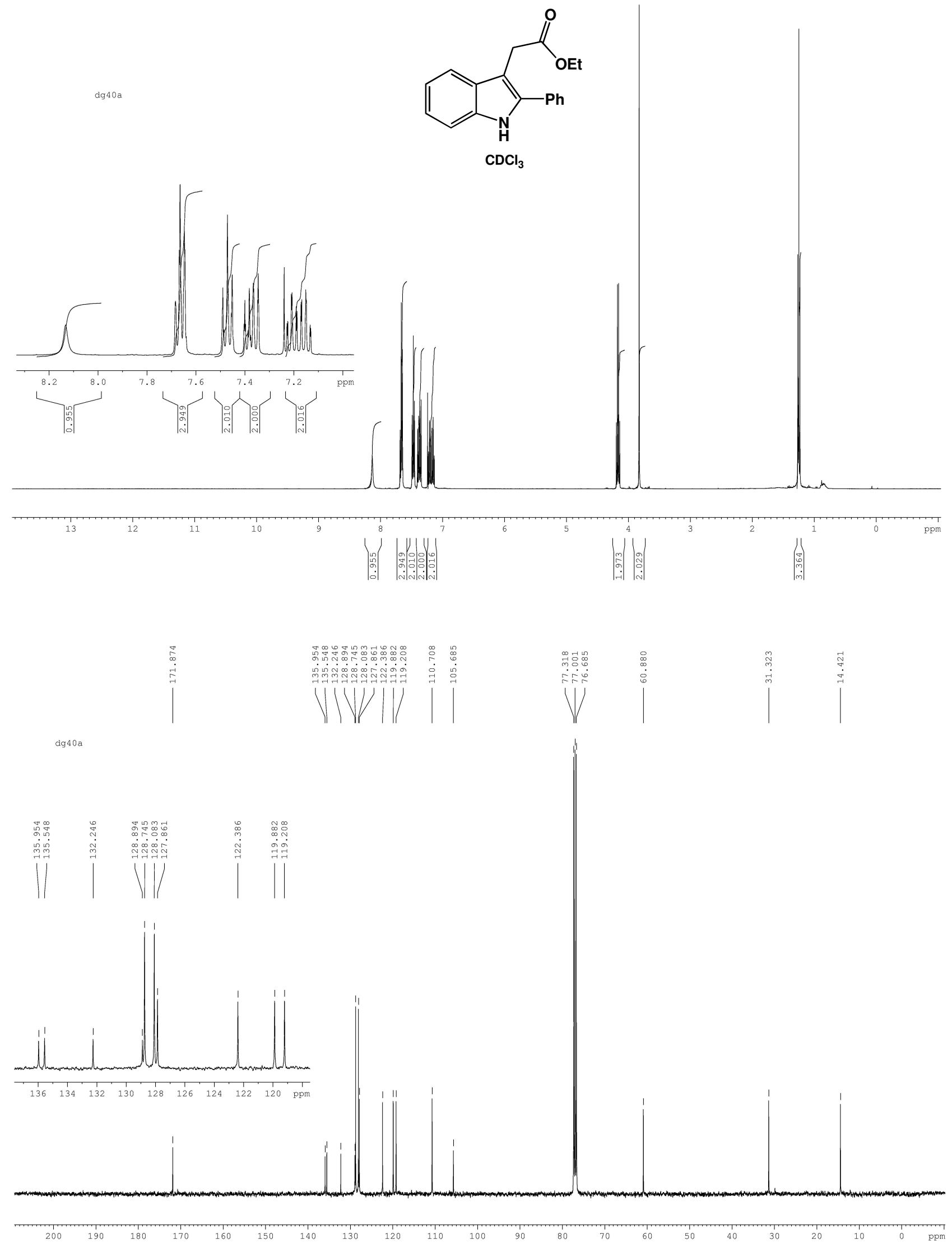



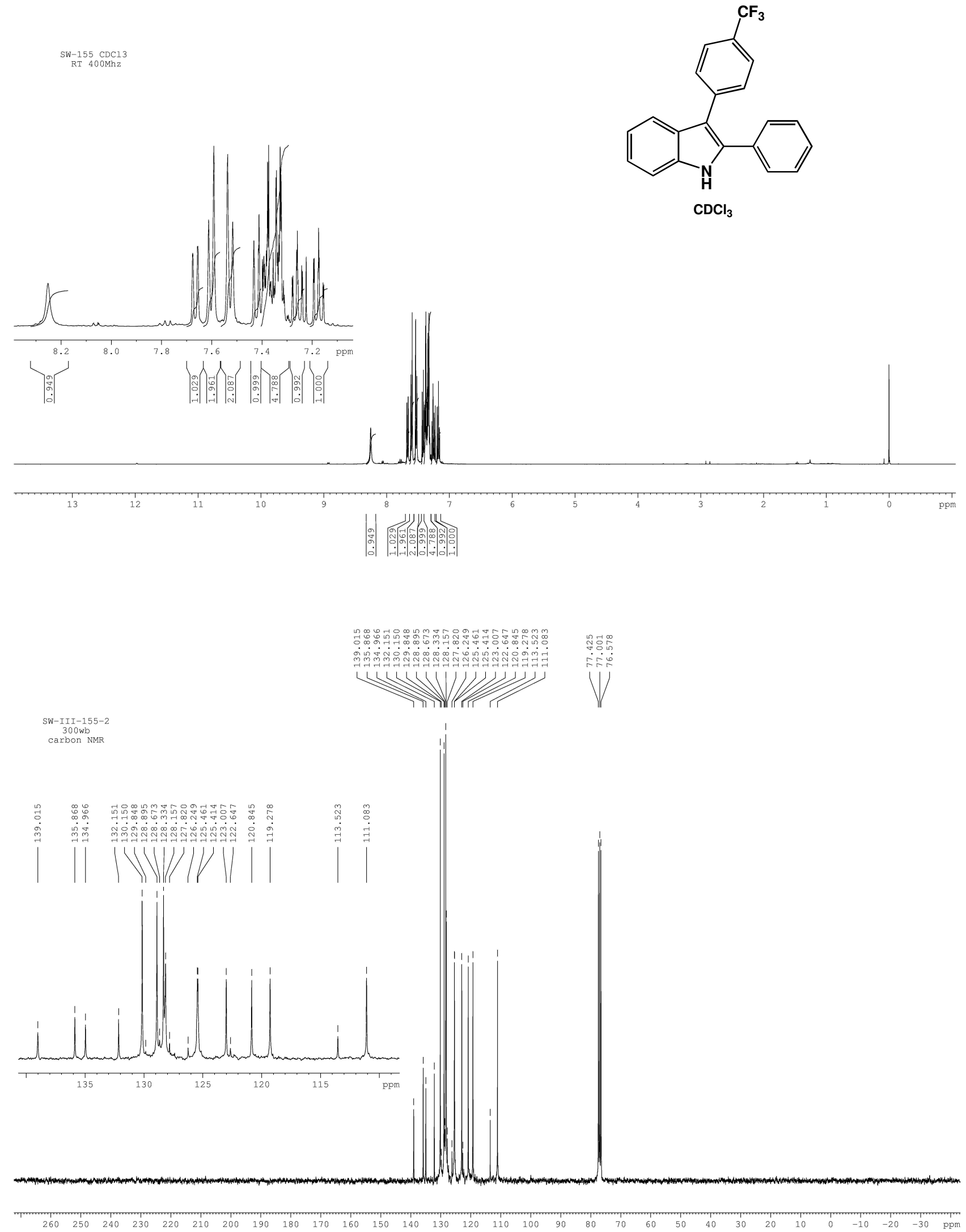

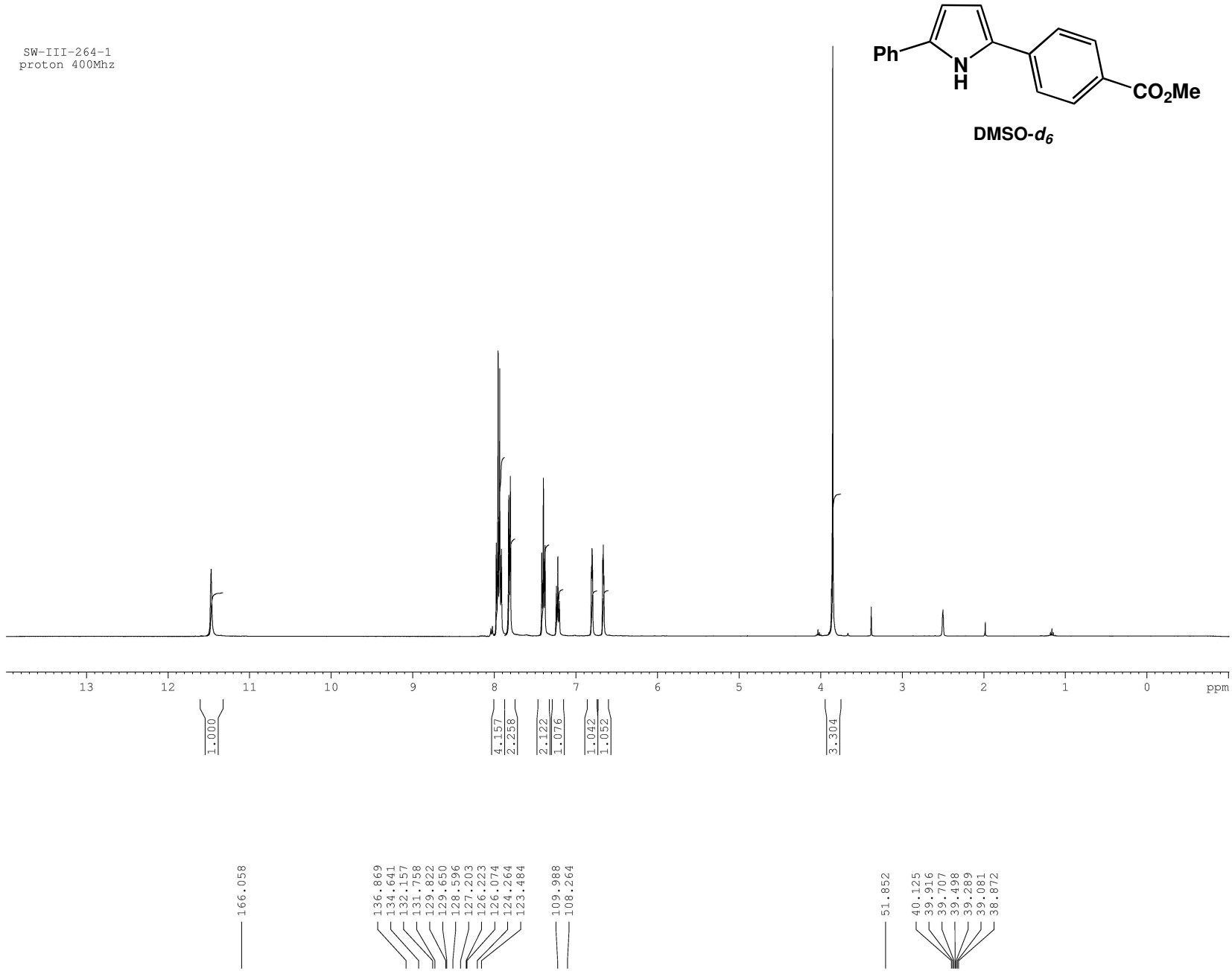

SW-III-264-2

carbon $100 \mathrm{Mh} z$
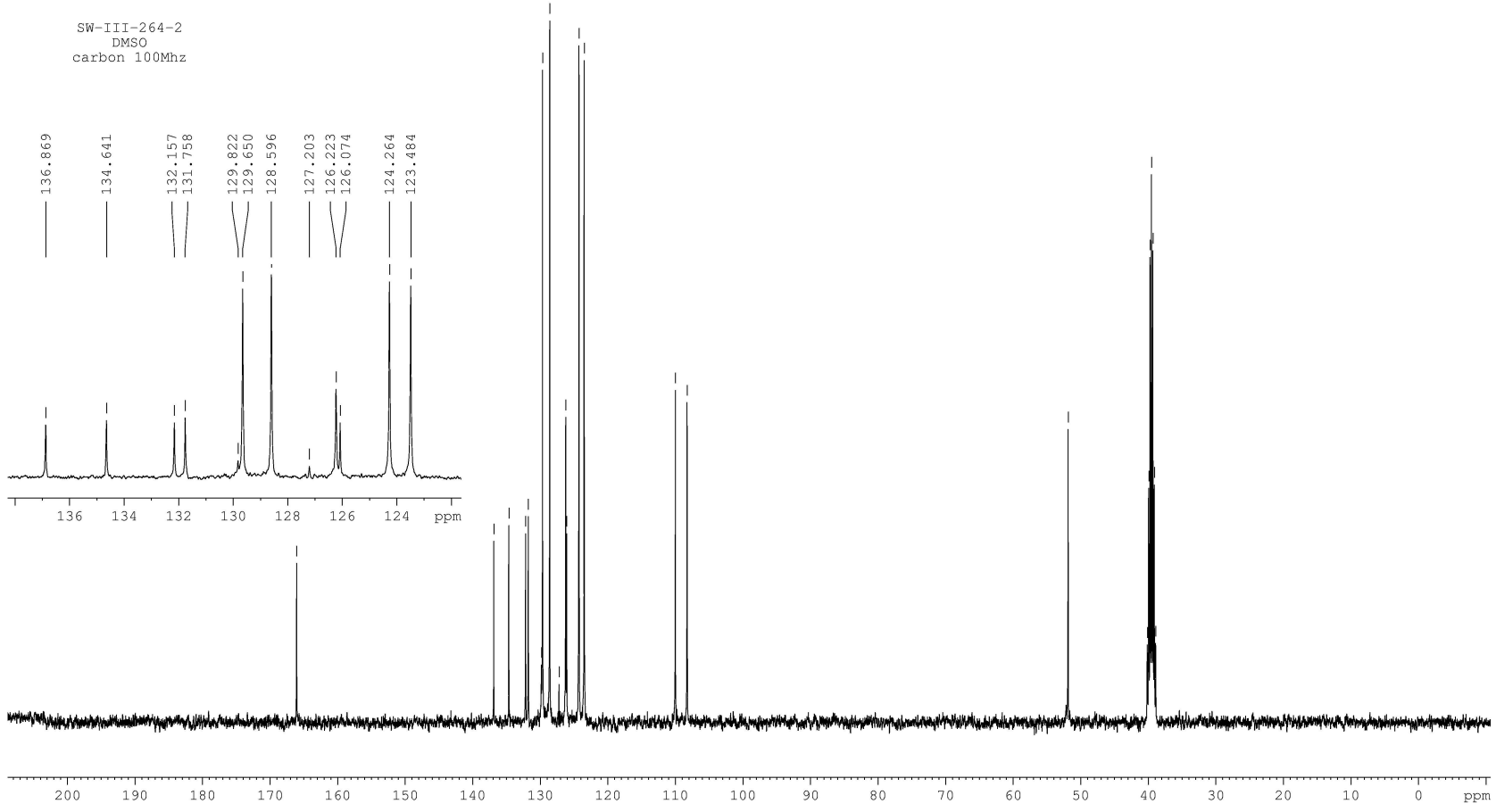


\section{References:}

${ }^{1}$ Eichberg, M. J.; Dorta, R. L.; Lamottke, K.; Vollhardt, K. P. Org. Lett. 2000, 2, 2479.

${ }^{2}$ Nicolaou, K. C.; Chen, D. Y.; Huang, X.; Ling, T.; Bella, M.; Snyder, S. A. J. Am. Chem. Soc. 2004, 126, 12888.

${ }^{3}$ Wang, X.; Lane, B. S.; Sames, D. J. Am. Chem. Soc. 2005, 127, 4996.

${ }^{4}$ Elzahraa, F.; Elbasil, S.; Elsayed, M.; Ghoneim, K. M.; Khalifa, M. Pharmazie 1979, 34, 12.

${ }^{5}$ Goodson, F. E.; Wallow, T. I.; Novak, B. M. J. Am. Chem. Soc. 1997, 119, 12441.

6 Schiavi, B.; Ahond, A.; Al-Mourabit, A.; Poupat, C.; Chiaroni, A.; Gaspard, C.; Potier, P. Tetrahedron 2002, 58, 4201.

${ }^{7}$ Hudkins, R. L.; Diebold, J. L.; Marsh, F. D. J. Org. Chem. 1995, 60, 6218.

${ }^{8}$ Caubere, C.; Caubere, P.; Ianelli, S.; Nardelli, M.; Jamartgregoire, B. Tetrahedron 1994, 50, 11903.

${ }^{9}$ Lane, B. S.; Sames, D. Org. Lett. 2004, 6, 2897.

${ }^{10}$ Knight, D. W.; Redfern, A. L.; Gilmore, J. J. Chem. Soc. Perkin. Trans. 1 2001, 2874.

${ }^{11}$ Mun, H. S.; Ham, W. H.; Jeong, J. H. J. Comb. Chem. 2005, 7, 130. 